\title{
DOES EARLY PSYCHOLOGICAL INTERVENTION PROMOTE RECOVERY FROM POSTTRAUMATIC STRESS?
}

\author{
Richard J. McNally, ${ }^{1}$ Richard A. Bryant, ${ }^{2}$ and Anke Ehlers ${ }^{3}$ \\ ${ }^{1}$ Harvard University; ${ }^{2}$ University of New South Wales, Sydney, Australia; and \\ ${ }^{3}$ Institute of Psychiatry, King's College London, London, United Kingdom
}

Summary-In the wake of the terrorist attacks at the World Trade Center, more than 9,000 counselors went to New York City to offer aid to rescue workers, families, and direct victims of the violence of September 11, 2001. These mental health professionals assumed that many New Yorkers were at high risk for developing posttraumatic stress disorder (PTSD), and they hoped that their interventions would mitigate psychological distress and prevent the emergence of this syndrome. Typically developing in response to horrific, life-threatening events, such as combat, rape, and earthquakes, PTSD is characterized by reexperiencing symptoms (e.g., intrusive recollections of the trauma, nightmares), emotional numbing and avoidance of reminders of the trauma, and hyperarousal (e.g., exaggerated startle, difficulty sleeping). People vary widely in their vulnerability for developing PTSD in the wake of trauma. For example, higher cognitive ability and strong social support buffer people against PTSD, whereas a family or personal history of emotional disorder heightens risk, as does negative appraisal of one's stress reactions (e.g., as a sign of personal weakness) and dissociation during the trauma (e.g., feeling unreal or experiencing time slowing down). However, the vast majority of trauma survivors recover from initial posttrauma reactions without professional help. Accordingly, the efficacy of interventions designed to mitigate acute distress and prevent long-term psychopathology, such as PTSD, needs to be evaluated against the effects of natural recovery. The need for controlled evaluations of early interventions has only recently been widely acknowledged.

Psychological debriefing - the most widely used method-has undergone increasing empirical scrutiny, and the results have been disappointing. Although the majority of debriefed survivors describe the experience as helpful, there is no convincing evidence that debriefing reduces the incidence of PTSD, and some controlled studies suggest that it may impede natural recovery from trauma. Most studies show that individuals who receive de-

Following the terrorist attacks at the World Trade Center, more than 9,000 grief and crisis counselors arrived in New York City to provide aid to families, rescue workers, and others

Address correspondence to Richard J. McNally, Department of Psychology, Harvard University, 33 Kirkland St., Cambridge, MA 02138; e-mail: rjm@wjh.harvard.edu. briefing fare no better than those who do not receive debriefing. Methodological limitations have complicated interpretation of the data, and an intense controversy has developed regarding how best to help people in the immediate wake of trauma.

Recent published recommendations suggest that individuals providing crisis intervention in the immediate aftermath of the event should carefully assess trauma survivors' needs and offer support as necessary, without forcing survivors to disclose their personal thoughts and feelings about the event. Providing information about the trauma and its consequences is also important. However, research evaluating the efficacy of such "psychological first aid" is needed.

Some researchers have developed early interventions to treat individuals who are already showing marked stress symptoms, and have tested methods of identifying those at risk for chronic PTSD. The single most important indicator of subsequent risk for chronic PTSD appears to be the severity or number of posttrauma symptoms from about 1 to 2 weeks after the event onward (provided that the event is over and that there is no ongoing threat).

Cognitive-behavioral treatments differ from crisis intervention (e.g., debriefing) in that they are delivered weeks or months after the trauma, and therefore constitute a form of psychotherapy, not immediate emotional first aid. Several controlled trials suggest that certain cognitive-behavioral therapy methods may reduce the incidence of PTSD among people exposed to traumatic events. These methods are more effective than either supportive counseling or no intervention.

In this monograph, we review risk factors for PTSD, research on psychological debriefing, recent recommendations for crisis intervention and the identification of individuals at risk of chronic PTSD, and research on early interventions based on cognitive-behavioral therapy. We close by placing the controversy regarding early aid for trauma survivors in its social, political, and economic context.

exposed to the mayhem of September 11, 2001 (Kadet, 2002). The assumption driving these well-intentioned efforts was that many New Yorkers were likely to develop posttraumatic stress disorder (PTSD) if they did not receive counseling soon after the trauma. Crisis Management International, a firm based in Atlanta, Georgia, sent 350 therapists, booking every room in one of New York's prominent hotels. The Church of Scientol- 
ogy sent 800 volunteers to provide "spiritual first aid" to Ground Zero rescue workers. Sites were quickly established throughout the city to accommodate the countless numbers of people expected to seek psychological help. Yet few people showed up. The demand for psychological services was far less than most experts had predicted (Kadet, 2002). ${ }^{1}$

Yehuda (2002a) — an eminent neuroscientist and PTSD expert-said that many therapists suspected that New Yorkers were in denial. A failure to seek counseling, they surmised, reflected avoidance behavior - a sign of PTSD. Accordingly, some therapists planned to approach citizens on the street and conduct quick sidewalk assessments and brief interventions for those presumably too avoidant to seek help on their own.

There were other reasons, besides denial of distress, why many New Yorkers did not avail themselves of proffered psychological services during the months following the attacks. People directly affected by the attacks - those who lost their loved ones or their jobs when the towers collapsed-were often too busy trying to put their lives back together to take time out for psychological counseling. And when they did seek professional assistance, it was often to obtain help in practical matters (e.g., getting death certificates for insurance purposes). ${ }^{2}$

Another explanation was that mental health experts had underestimated both the psychological resilience of New York's citizens and their extant sources of emotional support. Traumatized people often relied on family, friends, and church groups rather than seeking professional counseling. Not everyone exposed to trauma either needed or wanted psychological services.

Some experts predicted a surge in delayed-onset PTSD. People too preoccupied with urgent practical matters might fail to process the trauma shortly after it occurred, thereby suffering its effects many months later, once life began to return to normal. Anticipating an epidemic of delayed psychiatric prob-

1. Kadet arrived at the 9,000 figure by contacting representatives of the organizations whose members were offering counseling to people in New York City and asking them how many counselors each organization was supplying. She spoke to representatives from the International Critical Incident Stress Foundation, the Church of Scientology, the American Psychological Association, the Eye Movement Desensitization and Reprocessing Humanitarian Assistance Program, the Association of Traumatic Stress Specialists, Project Liberty, the Red Cross, the Green Cross, the City Department of Health Public Affairs, the International Society for Traumatic Stress Studies, the Center for Mental Health Services, and Crisis Management International (A. Kadet, personal communication, March 20,2003). These organizations offered various services to New Yorkers (e.g., psychological debriefing, eye movement desensitization and reprocessing). Kadet's conclusion about few people showing up for help was based on her interviews with clinicians who expressed surprise at the underutilization of their counseling services. Although articles in magazines and newspapers are seldom cited in scholarly journals, the facts uncovered by journalists, such as Kadet, appear in ordinary news media, not scientific publications, Therefore, we cite data from these sources when necessary.

2. Although providing assistance in obtaining death certificates might conceivably be considered a form of crisis intervention, it is certainly not counseling or psychotherapy. lems, authorities obtained \$23 million in federal funds to establish Project Liberty, a program designed to provide free counseling for New Yorkers (Kadet, 2002). An additional \$131 million was requested to pay for the 3,000 therapists hired by the project. Its director, April Naturale, predicted that one out of every four citizens of New York City would need therapy for emotional problems resulting from the attacks of September 11. However, as of March 2003, only 643,710 people had sought help through Project Liberty, whereas officials had expected to treat 2.5 million New Yorkers. As of May 2003, \$90 million of therapy funds remained unspent (Gittrich, 2003).

Concerns about preventing posttraumatic psychopathology, either immediate or delayed, motivated these massive intervention efforts. But do psychological interventions delivered shortly after traumatic events mitigate distress and prevent later problems, especially PTSD? In particular, do trauma-exposed people who receive psychological debriefing - the most popular intervention-experience fewer difficulties than do people who are not debriefed? Or does debriefing impede natural recovery from the effects of trauma? Answers to these questions are urgently needed because counselors trained to provide this service have become seemingly ubiquitous at the scene of diverse traumatic events (Deahl, 2000), from school shootings to natural disasters. If these interventions either have no effect or are harmful, are there promising alternatives that might prevent posttraumatic psychopathology? If so, should everyone exposed to trauma receive an intervention, or should only those individuals at high risk for psychopathology receive one? Finally, should resources be directed toward helping individuals who have already developed PTSD rather than toward attempts to prevent its emergence among those recently exposed to trauma?

Psychological debriefing is a generic term for a brief crisis intervention that is usually delivered within several days of a traumatic event and is designed in part to mitigate emotional distress and to prevent long-term psychopathology, especially significant symptoms of PTSD (Raphael \& Wilson, 2000). Its key elements are ventilating emotions about the trauma while discussing one's thoughts, feelings, and reactions with a trained professional who, in turn, provides psychoeducation about traumatic stress responses and attempts to normalize these reactions. Many people believe that it is better to talk about one's feelings than to "bottle them up inside," and any intervention requiring one to process and express emotions about a traumatic event within a supportive context would seem to be of unquestionable value. Indeed, many people are likely to assume that the quicker emotional first aid is provided, the less likely trauma-exposed people are to develop long-term psychological problems.

Despite the intuitive plausibility of these assumptions, psychological debriefing has sparked a heated international controversy that captured the attention of government policymakers, the media, and the general public after the recent terrorist attacks (e.g., Goode, 2001; Herbert et al., 2001). The controversy has grown as increasing numbers of studies have failed to confirm the efficacy of psychological debriefing as a method for at- 
tenuating posttraumatic distress (Raphael \& Wilson, 2000). Critics assert that public funds must be allocated only for methods shown to work; continuing to employ methods that are either inert or harmful will prevent clinical scientists from developing and testing methods that mitigate distress and prevent long-term psychiatric impairment.

In this review, we first briefly discuss PTSD and risk factors for the disorder. We then scrutinize the evidence regarding the efficacy of psychological debriefing, focusing on prevention of psychopathology, especially PTSD. We also discuss new research on cognitive-behavioral therapy (CBT) for recent-onset PTSD. In contrast to crisis-intervention methods delivered hours or days posttrauma (e.g., psychological debriefing), these new CBT intervention methods are applied weeks or months after the trauma. They are designed not to prevent disorder, but rather to help individuals whose symptoms have failed to abate within the first few weeks posttrauma. Finally, we close by considering the controversy in its larger social context.

\section{DEFINITION OF PTSD}

PTSD was first recognized as a psychiatric disorder in the third edition of the American Psychiatric Association's (APA) Diagnostic and Statistical Manual of Mental Disorders (1980). The current criteria, in the fourth edition of this manual (DSMIV; APA, 1994), define PTSD as a syndrome comprising three clusters of signs and symptoms: (a) repeated reexperience of the trauma (e.g., intrusive recollections of the event, nightmares); (b) emotional numbing (e.g., difficulty experiencing positive emotions) and avoidance of activities and stimuli reminiscent of the trauma; and (c) heightened arousal (e.g., exaggerated startle reflex, insomnia; see Table 1). Finally, a diagnosis of PTSD requires that these symptoms still be evident at least 1 month after trauma exposure and cause impairment or clinically significant distress.

\section{What Constitutes a Traumatic Event?}

Unlike the criteria for most DSM-IV disorders, those for PTSD require a specific etiologic event: exposure to a traumatic event. Regardless of how symptomatic a person might be, if the person has not been exposed to an event that counts as "traumatic," then the diagnosis cannot be assigned.

Trauma theorists originally conceptualized PTSD as a syndrome caused by exposure to extreme stressors occurring outside the boundary of everyday life-events likely to trigger marked distress in nearly everyone. Prior to revising the DSM, the DSM-IV PTSD committee discussed the pros and cons of revising the definition of a traumatic stressor. Some members worried that a such high threshold for classifying an experience as traumatic would exclude many people from receiving the diagnosis and the treatment they deserve. Others worried that broadening the definition would create other problems, both fo- rensic and scientific. If, for example, the definition were to certify any event as traumatic, as long as it was perceived as such, then the diagnosis would be prone to abuse in the courts. For example, a Michigan woman filed suit against her employer, claiming she developed PTSD as a result of repeatedly being exposed to practical jokes and foul language in the workplace (McDonald, 2003). She won, and the court awarded her \$21 million. Also, scientists worried that broadening the definition of a traumatic event would make it difficult to identify psychobiological mechanisms underlying symptoms arising from extremely diverse events.

As it turns out, the definition of traumatic stressor did broaden in DSM-IV and did emphasize the subjective perception of threat. To qualify as trauma exposed, one no longer needs to be a direct victim. As long as one is confronted with a situation that involves threat to the physical integrity of one's self or others and one experiences the emotions of fear, horror, or helplessness, then the experience counts as exposure to a PTSD-qualifying stressor. For two reasons, DSM-IV dropped the earlier requirement that a traumatic stressor had to be "an event that is outside the range of usual human experience" (APA, 1987, p. 250). First, it was unclear what constitutes "usual" human experience. Stressors outside this boundary for an affluent American might well be within the boundary of usual experience of someone in an impoverished, war-torn country in the Third World. Second, many events triggering PTSD, such as automobile accidents and criminal assaults, are far from uncommon.

\section{The Psychological Impact of the September 11 Terrorist Attacks}

The broadened definition of a traumatic event is relevant to concerns about people developing PTSD symptoms following indirect exposure to the events of September 11, such as watching television footage of the attacks on the World Trade Center. Given that one no longer had to be the direct victim (or even direct witness) of trauma-having been "confronted with" a terrible event on television now qualified as a DSM-IV traumatic stressor-concerns arose about posttraumatic responses throughout the country. For example, the RAND Corporation interviewed a representative sample of 560 adults throughout the United States on the weekend after the attacks, concluding that $44 \%$ of Americans "had substantial symptoms of stress" (Schuster et al., 2001, p. 1507), and ominously warning that the psychological effects of terrorism "are unlikely to disappear soon" (p. 1511) and that "clinicians should anticipate that even people far from the attacks will have trauma-related symptoms" (p. 1512). The researchers arrived at these conclusions as follows. Respondents were asked whether they had experienced any of five symptoms "since Tuesday" (i.e., September 11, 2001) and rated each symptom on a 5-point scale ranging from 1 (not at all) to 5 (extremely). Respondents qualified as "substantially stressed" if they assigned a rating of 
Table 1. Diagnostic criteria for posttraumatic stress disorder

A. The person has been exposed to a traumatic event in which both of the following were present:

(1) the person experienced, witnessed, or was confronted with an event or events that involved actual or threatened death or serious injury, or a threat to the physical integrity of self or others

(2) the person's response involved intense fear, helplessness, or horror. Note: In children, this may be expressed instead by disorganized or agitated behavior

B. The traumatic event is persistently reexperienced in one (or more) of the following ways:

(1) recurrent and intrusive distressing recollections of the event, including images, thoughts, or perceptions. Note: In young children, repetitive play may occur in which themes or aspects of the trauma are expressed.

(2) recurrent distressing dreams of the event. Note: In children, there may be frightening dreams without recognizable content.

(3) acting or feeling as if the traumatic event were recurring (includes a sense of reliving the experience, illusions, hallucinations, and dissociative flashback episodes, including those that occur on awakening or when intoxicated). Note: In young children, traumaspecific reenactment may occur.

(4) intense psychological distress at exposure to internal or external cues that symbolize or resemble an aspect of the traumatic event

(5) physiological reactivity on exposure to internal or external cues that symbolize or resemble an aspect of the traumatic event

C. Persistent avoidance of stimuli associated with the trauma and numbing of general responsiveness (not present before the trauma), as indicated by three (or more) of the following:

(1) efforts to avoid thoughts, feelings, or conversations associated with the trauma

(2) efforts to avoid activities, places, or people that arouse recollections of the trauma

(3) inability to recall an important aspect of the trauma

(4) markedly diminished interest or participation in significant activities

(5) feeling of detachment or estrangement from others

(6) restricted range of affect (e.g., unable to have loving feelings)

(7) sense of a foreshortened future (e.g., does not expect to have a career, marriage, children, or a normal life span)

D. Persistent symptoms of increased arousal (not present before the trauma), as indicated by two (or more) of the following:

(1) difficulty falling or staying asleep

(2) irritability or outbursts of anger

(3) difficulty concentrating

(4) hypervigilance

(5) exaggerated startle response

E. Duration of the disturbance (symptoms in Criteria B, C, and D) is more than 1 month.

F. The disturbance causes clinically significant distress or impairment in social, occupational, or other important areas of functioning. Specify if:

Acute: if duration of symptoms is less than 3 months

Chronic: if duration of symptoms is 3 months or more

Specify if:

With Delayed Onset: if onset of symptoms is at least 6 months after the stressor

Note. From Diagnostic and Statistical Manual of Mental Disorders, fourth edition, by the American Psychiatric Association, 1994, pp. 427-429.

Copyright 1994 by the American Psychiatric Association. Reprinted with permission of the author.

at least 4 (quite $a$ bit) to one of the five symptoms. For example, anyone who acknowledged experiencing "quite a bit" of anger at Osama bin Laden qualified as substantially stressed. Another survey conducted in November 2001 revealed that $28 \%$ of adults throughout America had been offered counseling by their employers to cope with emotional effects of September 11 (Kadet, 2002).

Some authors have questioned the validity of these kinds of studies. As Wakefield and Spitzer (2002) have argued, such surveys often classify normal, expectable emotional reactions as symptoms of mental disorder. Moreover, many psychological, emotional, and physical reactions_-"symptoms"-are nonspecific and not necessarily indicative of serious psychiatric illness. For example, consider a resident of New York City who was working downtown on the day of the terrorist attacks, and who later reports difficulty falling asleep, difficulty concentrating, and irritability. Although these qualify as "symptoms of PTSD," each may arise for reasons unrelated to the attacks. Likewise, it would be misleading to refer to nonspecific medical symptoms, such as cough and fatigue, as symptoms of bacterial pneumonia in the absence of additional evidence (e.g., a culture).

Other surveys suggest that stress reactions in the wake of September 11 in many cases may have been temporary, normal reactions. For example, Galea et al. (2002) surveyed residents of New York City to gauge their response to the terrorist attacks. Five to 8 weeks after the attacks, $7.5 \%$ of a random sample of adults living south of 110th Street in Manhattan had developed PTSD, and of those living south of Canal Street (near the World Trade Center), 20\% had PTSD. In February 
2002, Galea's group did a follow-up study on another group of adults living south of 110th Street, and found that only $1.7 \%$ of the sample had PTSD related to the attacks (Galea, Boscarino, Resnick, \& Vlahov, in press). The $7.5 \%$ rate obtained within weeks of the attacks may have reflected temporary distress rather than mental illness. This study, like many others in the field (e.g., Kessler, Sonnega, Bromet, Hughes, \& Nelson, 1995), demonstrates that most people are resilient and recover from early posttrauma symptoms. The authors of another postSeptember 11 survey of New Yorkers concluded that "those with severe symptoms were far fewer than what we expected, given the magnitude and amount of personal exposure to this disastrous event" (DeLisi et al., 2003, p. 782).

\section{Exposure to Trauma and PTSD}

Many therapists have conceptualized PTSD as a normal, expectable reaction to an extraordinary stressor, despite its classification as a mental disorder. The traumatic event itself has been awarded overriding causal significance in producing PTSD; personal vulnerability factors have been minimized. These assumptions have been increasingly questioned in recent years, however (Yehuda \& McFarlane, 1995). Epidemiological studies have shown that many American adults have been exposed to DSM-defined traumatic stressors, such as physical assault, rape, or automobile accidents, yet few of them have developed PTSD. The National Comorbidity Survey revealed that $60.7 \%$ of randomly sampled adults reported exposure to DSM traumatic stressors (Kessler et al., 1995). But of these trauma-exposed people, only $20.4 \%$ of the women and $8.2 \%$ of the men had ever developed PTSD. Among adults living in metropolitan Detroit, $89.6 \%$ reported exposure to DSM traumatic stressors, yet only $13 \%$ of the women and $6.2 \%$ of the men had developed PTSD (Breslau, Davis, Andreski, \& Peterson, 1991).

Among traumatic stressors, those involving intentional acts of violence are especially likely to produce PTSD (Yehuda, 2002 b). In one epidemiological survey, PTSD developed in $11.6 \%$ of respondents who had experienced a sudden injury or accident, but in $22.6 \%$ of those who had experienced physical assault and in $80 \%$ of female rape victims (Breslau et al., 1991). In another study, the single most frequent event causing PTSD was learning about the unexpected death of a loved one; $26.5 \%$ of female cases and $38.5 \%$ of male cases of PTSD were attributed to this very common event (Breslau, Chilcoat, Kessler, Peterson, \& Lucia, 1999). (Being "confronted with an event" qualifies as a PTSD-inducing traumatic stressor, according to DSM-IV; APA, 1994, p. 427. Thus, individuals who, for example, receive a phone call informing them of the unexpected death of a loved one qualify as having been exposed to a traumatic stressor.) Breslau, Chilcoat, Kessler, Peterson, and Lucia found that men are more likely to be exposed to trauma than women, but that trauma-exposed women develop PTSD at twice the rate as do trauma-exposed men, mainly because exposure to criminal violence precipitates PTSD at a much higher rate in women than in men $(35.7 \%$ vs. $6 \%)$.

\section{The Time Course of Posttrauma Symptoms}

Most people recover from acute symptoms within 3 months posttrauma, even if they do not receive any treatment (e.g., Kessler et al., 1995). For example, Riggs, Rothbaum, and Foa (1995) reported that $71 \%$ of women and $50 \%$ of men met symptomatic criteria for PTSD (the requirement of 1-month duration was waived) approximately 19 days after a nonsexual assault. Four months posttrauma, the rate of PTSD had dropped to $21 \%$ for women and $0 \%$ for men. This research group also reported that $94 \%$ of rape victims interviewed an average of 2 weeks posttrauma met criteria for (acute) PTSD; 11 weeks later, the rate dropped to $47 \%$ (Rothbaum, Foa, Riggs, Murdock, \& Walsh, 1992). About half of trauma survivors who are still symptomatic at 3 months recover over the next few years (Ehlers, Mayou, \& Bryant, 1998; Kessler et al., 1995; Schnyder \& Moergeli, in press).

\section{RISK FACTORS FOR PTSD}

Researchers have endeavored to identify variables that heighten risk for PTSD, studying both who is most likely to be exposed to trauma and who among the trauma exposed is most likely to develop the disorder (Brewin, Andrews, \& Valentine, 2000; Yehuda, 1999).

\section{Risk Factors for Trauma Exposure}

The most important risk factor for PTSD is, of course, exposure to trauma. People vary considerably in this risk. Certain occupations clearly increase risk (e.g., soldier, firefighter). Some studies suggest that the people who choose these occupations possess other characteristics (e.g., psychological hardiness) that counteract the risk of PTSD that exposure to trauma entails.

For example, North et al. (2002) assessed 176 male firefighters approximately 34 months after they had done rescue and recovery work at the site of the Oklahoma City terrorist bombing. The rate of disaster-related PTSD (13\%) was significantly lower among the firefighters than among 88 male primary victims of the bombing itself (23\%). Among firefighters with any psychiatric disorder after the bombing, $82 \%$ had preexisting psychopathology. Unfortunately, North et al. were unable to interview all of the firefighters, and volunteers for the study constituted less than $25 \%$ of those working at the site.

North et al. (2002) speculated that psychological preparedness for dealing with gruesome aspects of firefighting may have been one variable fostering resilience among the men who worked at the Oklahoma bombing site. Similarly, Başoğlu, 
Mineka, Paker, Livanou, and Gök (1997) reported that psychological preparedness for trauma buffers individuals against subsequent symptoms. These researchers found significantly lower rates of current PTSD among left-wing Turkish political activists who had been tortured by the military regime than among nonactivist Turks who had been arrested for nonpolitical crimes and tortured (18\% vs. $58 \%$ ). These findings are especially striking because the activists had been exposed to more torture than the nonactivists. Combining both activist and nonactivist groups for further analyses, Başoğlu et al. found that the more psychologically prepared victims were (e.g., knowing about torture methods, being aware that torture often followed arrest, being trained in stoicism techniques), the less severe were their torture-related PTSD symptoms.

Realizing that severe stressors often do not strike people at random, Breslau's research group endeavored to identify risk factors for traumatic exposure in the general population. In a retrospective study, they found that adults reporting having had childhood conduct problems were more likely than other adults to be exposed to trauma in adulthood (Breslau et al., 1991). People reporting mental illness in their family members were also at heightened risk for exposure to trauma. In their subsequent prospective epidemiological study, Breslau, Davis, and Andreski (1995) found that higher scores on questionnaire measures of extraversion and neuroticism were associated with increased likelihood of subsequent exposure to traumatic events. Men were at greater risk than women; individuals lacking a college degree were at greater risk than college graduates; and Blacks were at greater risk than Whites.

\section{Risk Factors for PTSD Among People Exposed to Trauma}

Among people exposed to traumatic events, what factors are associated with increased risk for PTSD? These factors might include both variables operative prior to the trauma (e.g., high vs. low cognitive ability) and variables that come into play posttrauma (e.g., high vs. low levels of social support).

\section{Cross-sectional studies}

Some cross-sectional studies on people with PTSD have revealed correlates of the disorder that constitute plausible risk factors, even though these variables were measured after individuals had developed PTSD. People who are already suffering from anxiety or mood disorders are at heightened risk for PTSD, as are those with a family history of these disorders (Breslau et al., 1991). Relative to other trauma-exposed adults, those with PTSD report higher rates of having been sexually (Nishith, Mechanic, \& Resick, 2000) or physically (Breslau, Chilcoat, Kessler, \& Davis, 1999) abused during childhood. Retrospective reports of instability in one's family during childhood are likewise associated with PTSD (King, King, Foy, \& Gudanowski, 1996).
Cognitive ability is related to risk for PTSD among people exposed to trauma. For example, McNally and Shin (1995) found that lower intelligence was associated with greater severity of PTSD symptoms among Vietnam veterans even after controlling for the extent of combat exposure (measured by the veterans' self-reports). Other researchers have replicated this finding (Silva et al., 2000; Vasterling, Brailey, Constans, Borges, \& Sutker, 1997; Vasterling et al., 2002). For example, studying inner-city children and adolescents, Silva et al. (2000) found that IQ was the best predictor of resilience against PTSD among those exposed to trauma (e.g., witnessing violence, being sexually abused). Of those with above-average IQ scores, $67 \%$ had neither PTSD nor subthreshold PTSD. Of those with below-average IQ scores, only $20 \%$ had no PTSD symptoms. Note, however, that in these studies, researchers administered tests of cognitive ability among people who had already been exposed to trauma and who had already (in many cases) developed PTSD.

Neuroticism-a personality trait reflecting proneness to experience negative emotions (irritability, anxiety, depression)is higher among trauma-exposed people with PTSD than among those without the disorder (e.g., Breslau et al., 1991; McFarlane, 1989). Unfortunately, it is unclear whether heightened neuroticism results from trauma (or PTSD), is a vulnerability factor for PTSD, or both.

Some retrospectively assessed risk factors tap variables apparently linked to the posttrauma environment rather than pretrauma antecedents. For example, self-reported low social support is associated with PTSD among Vietnam combat veterans (e.g., Boscarino, 1995; Keane, Scott, Chavoya, Lamparski, \& Fairbank, 1985) and among civilians (see meta-analysis by Brewin et al., 2000). (Meta-analysis is a statistical technique for combining the results of similar studies, hence enhancing the reliability of any conclusion about the hypothesis under consideration.) Thus, people who are exposed to trauma, but who lack social support, may be at heightened risk for developing PTSD. Stated differently, a supportive posttrauma environment might hasten reduction in acute symptoms, thereby reducing risk for PTSD. Unfortunately, it is difficult to tell what mechanism is responsible for the association between low social support and risk of PTSD. It may be that posttrauma symptoms, such as anger outbursts and emotional withdrawal, alienate potential sources of social support. In addition, impoverished social-support networks make it difficult for people to overcome the effects of trauma. Nevertheless, negative perceptions of other people's responses (e.g., "I feel that other people are ashamed of me now") predict PTSD beyond what can be predicted from initial symptom levels (Dunmore, Clark, \& Ehlers, 2001).

\section{Prospective studies}

The ideal method for identifying risk factors for PTSD is to take pretrauma measurements on a large number of individu- 
als, perhaps those likely to encounter traumatic events (e.g., firefighters, military personnel), and to follow them over time. One can then identify those who are exposed to trauma and determine the putative risk factors that seem to predict who develops the disorder and who does not, as well as who recovers. Such prospective, longitudinal studies are expensive and therefore difficult to conduct, however. Another approach is to comb archival records of trauma-exposed individuals and identify variables, measured pretrauma, that correlate with PTSD.

Studying predeployment military archives of inductees who later fought in Vietnam, researchers found that those who developed PTSD had reported more school difficulties, had lower arithmetic aptitude, and (paradoxically) had lower heart rate than did combat veterans who did not develop PTSD (Pitman, Orr, Lowenhagen, Macklin, \& Altman, 1991). Because of multiple statistical comparisons, Pitman et al. urged readers to interpret these significant differences as "trends" (p. 418). Schnurr, Friedman, and Rosenberg (1993) obtained premilitary scores from the Minnesota Multiphasic Personality Inventory (MMPI; a self-report measure of normal and abnormal personality variation) for a group of Dartmouth College undergraduates who later served in Vietnam. After controlling for extent of combat exposure, Schnurr et al. found that elevations on several MMPI scales (Hypochondriasis, Psychopathic Deviate, Paranoia, Femininity) predicted PTSD symptoms. In another study, Bramsen, Dirkzwager, and van der Ploeg (2000) found that predeployment negativism - a personality trait similar to neuroticism-predicted subsequent PTSD symptoms among Dutch peacekeepers serving in the former Yugoslavia.

Two prospective studies of Vietnam veterans and Israeli soldiers indicate that above-average intelligence may buffer people against the traumatic effects of stressors (Kaplan et al., 2002; Macklin et al., 1998). In both studies, higher precombat IQ scores predicted lower risk for PTSD following combat. Lower precombat intelligence predicted greater severity of PTSD symptoms at the time of the studies (Macklin et al., found that this was true even after controlling statistically for amount of self-reported combat exposure). Macklin et al. found no significant association between severity of PTSD symptoms at the time of the study and change in IQ score since predeployment. In other words, the stress of chronic PTSD did not lower scores on IQ tests taken at the time of the study.

\section{Peritraumatic risk factors}

Another approach to studying risk factors is to identify peritraumatic responses-those occurring during the trauma itself or shortly after it-that predict later PTSD. Studies that take this approach are longitudinal in the sense that traumatized people are followed over time, but they are not truly prospective because measurements are taken after trauma has occurred. They are prospective in the sense that measurements are taken shortly after the event, before a month has elapsed (the duration requirement for PTSD).
Researchers have tested whether the severity of symptoms at various times posttrauma is correlated with subsequent PTSD. Symptom severity in the first few days following the trauma fails to predict later PTSD (e.g., Shalev, 1992), whereas greater symptom severity from 1 to 2 weeks posttrauma and onward correlates highly with subsequent symptom severity (e.g., Harvey \& Bryant, 1998b; Murray, Ehlers, \& Mayou, 2002; Shalev, Freedman, Peri, Brandes, \& Sahar, 1997). For example, an Israeli study of survivors of motor vehicle accidents indicated that greater severity of PTSD symptoms assessed 1 week after the accident predicted greater risk of a diagnosis of PTSD 1 year later (Koren, Arnon, \& Klein, 1999). However, as Shalev et al. pointed out, low initial symptom levels are better at predicting a favorable outcome than high initial symptom levels are at predicting PTSD.

Several studies have suggested that self-reported peritraumatic dissociation (e.g., feeling unreal or experiencing time slowing down during the trauma) predicts subsequent PTSD among trauma survivors. Indeed, a recent meta-analysis indicated that peritraumatic dissociation was the single best predictor of PTSD $(r=.35)$ among trauma-exposed individuals (Ozer, Best, Lipsey, \& Weiss, 2003). Unfortunately, Ozer et al. failed to include cognitive ability in their meta-analysis, and both cross-sectional (e.g., Vasterling et al., 1997- $r=-.37$ ) and prospective (e.g., Macklin et al., 1998- $r=-.45$ ) studies have shown that the predictive power of cognitive ability is at least as great as that of peritraumatic dissociation.

Some researchers have investigated possible links between specific symptoms of peritraumatic dissociation and risk for subsequent PTSD. Among Israeli civilians exposed to motor vehicle accidents, terrorist attacks, and other traumatic events, a sense that what was happening was unreal (derealization) and a sense of time distortion (things happening in slow motion) measured 1 week posttrauma-predicted who met criteria for PTSD 6 months later (Shalev, Peri, Canetti, \& Schreiber, 1996). In another study, depersonalization (feeling disconnected from one's body), emotional numbing, motor restlessness, and a sense of reliving the trauma-measured within 1 month after the trauma-predicted later PTSD among survivors of automobile accidents (Harvey \& Bryant, 1998b). However, Murray et al. (2002) found that although peritraumatic dissociation measured within $24 \mathrm{hr}$ or 1 week of the trauma predicted PTSD 6 months after a motor vehicle accident, persistent dissociation at 4 weeks was a more powerful predictor.

It remains unclear whether dissociation in the aftermath of the trauma predicts PTSD over and above what can be predicted from symptoms of reexperiencing, avoidance, and hyperarousal. Brewin, Andrews, Rose, and Kirk (1999) and G.N. Marshall and Schell (2002) found no evidence that peritraumatic dissociation is an independent and unique predictor of subsequent PTSD severity, whereas Murray et al. found that dissociation improved the prediction of subsequent PTSD severity. Among individuals exposed to community violence, peritraumatic dissociation failed to predict later PTSD symp- 
toms once Marshall and Schell controlled for initial severity of PTSD symptoms. This study also highlighted the problems associated with earlier studies that required trauma survivors to recall their levels of peritraumatic association at a later time, because Marshall and Schell found that later recall of peritraumatic dissociation was often inaccurate.

Overall, there are mixed findings concerning the extent to which acute dissociative symptoms predict PTSD. In several studies, peritraumatic dissociation was a powerful predictor of subsequent PTSD (e.g., Ehlers et al., 1998; Koopman, Classen, \& Spiegel, 1994; Murray et al., 2002; Shalev, Freedman, et al., 1998; see also the meta-analysis of Ozer et al., 2003). However, peritraumatic dissociation failed to predict PTSD in other prospective studies (e.g., Dancu, Riggs, Hearst-Ikeda, Shoyer, \& Foa, 1996; G.N. Marshall \& Schell, 2002). These findings are consistent with other evidence that peritraumatic dissociation does not necessarily result in later psychiatric disorders (see Harvey \& Bryant, 2002). For example, survivors of harrowingly close brushes with death often report having experienced intense dissociation (e.g., depersonalization, time seeming to slow down), but they seldom develop later psychiatric problems (Noyes \& Kletti, 1976, 1977). Indeed, among these survivors, "many commented that they had been without frightening dreams or anxiety after their accidents and also that they had not found memory of the accident disturbing" (Noyes \& Kletti, 1976 , p. 26). Because these close brushes with death produce so "little traumatic aftermath" (Noyes \& Kletti, 1977, p. 382), Noyes and Kletti concluded that peritraumatic depersonalization is an adaptive mechanism.

How can one make sense of the mixed findings about the capacity of peritraumatic dissociation to predict subsequent PTSD? There are several explanations available. One possibility is that peritraumatic dissociation leads to later PTSD because the dissociation is associated with other known risk factors. Keane, Kaufman, and Kimble (2001) noted the evidence suggesting that a history of childhood trauma is associated with subsequent dissociation (Spiegel \& Cardeña, 1991), and raised the possibility that peritraumatic dissociation may be linked to PTSD because of its association with childhood trauma, which is a strong risk factor for subsequent PTSD. Keane et al. proposed that more rigorous testing of the link between peritraumatic dissociation and PTSD will be achieved by including dissociative reactions at the time of the recent precipitating trauma in mathematical models that test the relative contributions of pretrauma, peritraumatic, and posttrauma factors. The possible association of acute dissociation with distinct vulnerability factors may explain, to some degree, the discrepant findings about the relationship of peritraumatic dissociation and PTSD.

A related possibility is that dissociation influences the development of PTSD in only a subset of trauma-exposed individuals. Some theorists have proposed diathesis-stress models according to which only people who possess dissociative tendencies may respond to trauma with dissociative reactions
(Butler, Duran, Jasiukaitis, Koopman, \& Spiegel, 1996). Consistent with this notion is evidence that people who display dissociative reactions following trauma are more hypnotizable (hypnotizability is strongly correlated with dissociative tendencies) than people who develop acute stress reactions without dissociative symptoms (Bryant, Guthrie, \& Moulds, 2001). Although both groups may have high risk for developing PTSD, only the subset of people who possess dissociative tendencies appear to respond with acute dissociative symptoms. This pattern may explain why acute dissociation is not uniformly found to be a strong predictor of subsequent PTSD. The proposal that some people will develop PTSD after having acute dissociative symptoms and others will develop PTSD without this initial reaction points to different pathways of PTSD development. Consistent with the view that there are multiple pathways to PTSD is evidence that dissociation is a stronger predictor of subsequent PTSD in women than in men (Bryant \& Harvey, 2003).

Another possibility is that the influence of peritraumatic dissociation on subsequent PTSD may be mediated by how individuals appraise their dissociative reactions. Cognitive theorists posit that PTSD develops and is maintained by catastrophic interpretations of both the traumatic event and the individual's resultant responses (Ehlers \& Clark, 2000). People who are likely to develop posttraumatic psychopathology exaggerate the probability of future negative events occurring (Smith \& Bryant, 2000; Warda \& Bryant, 1998). Further, negative appraisals in the initial period after trauma exposure predict subsequent PTSD (Engelhard, van den Hout, Arntz, \& McNally, 2002), as do catastrophic attributions of responsibility for a trauma in this period (Andrews, Brewin, Rose, \& Kirk, 2000; Delahanty et al., 1997). Negative appraisals of peritraumatic dissociation may predict subsequent PTSD better than do dissociative reactions themselves. For example, an individual who interprets emotional numbing as a normal response to an assault may be less distressed than an individual who interprets numbing as a sign of madness.

For a range of traumas, how distressed a victim was during the trauma or how threatened the person felt (e.g., whether the person believed that he or she was about to die) often has predicted PTSD better than objective measures of stressor magnitude, such as the extent of bodily injury (e.g., Ehlers et al., 1998; Perry, Difede, Musngi, Frances, \& Jacobsberg, 1992; Schnyder, Moergeli, Klaghofer, \& Buddeberg, 2001). The higher the distress or perceived threat, the more severe PTSD symptoms were likely to be.

The conclusions that people draw from the trauma can be distorted and can contribute to the subsequent distress, making it difficult for survivors to put the event in the past. For example, rape victims may feel very ashamed and blame themselves for their attacks; accident survivors may think they are incompetent because they did not prevent the crash from happening; and assault survivors may feel that there is something about them that will make it very likely that they will be attacked 
again. Such highly idiosyncratic, excessively negative appraisals distinguish well between trauma survivors with and without PTSD currently (Foa, Ehlers, Clark, Tolin, \& Orsillo, 1999), as well as between survivors who are and are not likely to develop PTSD in the future. For example, Dunmore et al. (2001) found that negative appraisals assessed within 4 months posttrauma predicted PTSD symptom severity among assault survivors 6 months and 9 months posttrauma.

Finally, not only does appraisal of the stressor affect its pathogenic impact, but appraisal of acute stress symptoms themselves may influence whether chronic PTSD develops (Ehlers \& Clark, 2000). Catastrophic appraisal of symptoms as harbingers of impending psychosis or indicants of moral weakness increase risk for PTSD (e.g., Dunmore et al., 2001; Ehlers et al., 1998). For example, appraisal of intrusive thoughts as meaning that one is about to lose one's mind, rather than as a temporary and expectable response to trauma, may foster attempts to suppress intrusive thoughts, which in turn may lead to a paradoxical increase in their frequency. As this example shows, excessively negative appraisals of the trauma and its consequences motivate trauma survivors to engage in behaviors that maintain the problem. Likewise, some safety behaviors are strong predictors of PTSD. These behaviors include taking excessive precautions (e.g., Dunmore et al., 2001), excessively avoiding trauma reminders (e.g., Harvey \& Bryant, 1998a), and ruminating about the trauma and its effects on one's life (e.g., Murray et al., 2002).

\section{ACUTE STRESS DISORDER}

The diagnosis acute stress disorder (ASD) made its first appearance in DSM-IV. ASD arises from the same set of traumatic stressors deemed capable of causing PTSD, and is characterized by many of the same symptoms (see Table 2). According to DSM-IV, ASD can occur after exposure to a threatening event, and is diagnosed if the individual exhibits at least three dissociative symptoms, one reexperiencing symptom, marked avoidance, and marked hyperarousal. ASD differs from PTSD in two critical ways. First, the disturbance must last for a minimum of 2 days and a maximum of 4 weeks (after which time a diagnosis of PTSD could be made). Second, the ASD criteria emphasize dissociative reactions. According to DSM-IV, the diagnosis of ASD requires the presence of at least three of the following dissociative symptoms: a sense of emotional numbing or detachment, reduced awareness of one's surroundings, derealization, depersonalization, and amnesia for aspects of the traumatic event. In contrast, the PTSD criteria do not require the individual to display dissociative symptoms.

ASD advocates advanced three arguments for including the disorder in DSM-IV. First, although a diagnosis of PTSD required 1 month of persistent symptoms (so that transient stress reactions would not be classified as pathological), it is inhumane to make highly distressed survivors wait 1 month before diagnosing and treating a severe stress reaction. Indeed, doctors would not wait 1 month to diagnose and treat a broken

Table 2. Diagnostic criteria for acute stress disorder

A. The person has been exposed to a traumatic event in which both of the following were present:

(1) the person experienced, witnessed, or was confronted with an event or events that involved actual or threatened death or serious injury, or a threat to the physical integrity of self or others

(2) the person's response involved intense fear, helplessness, or horror

B. Either while experiencing or after experiencing the distressing event, the individual has three (or more) of the following dissociative symptoms:

(1) a subjective sense of numbing, detachment, or absence of emotional responsiveness

(2) a reduction in awareness of his or her surroundings (e.g., "being in a daze")

(3) derealization

(4) depersonalization

(5) dissociative amnesia (i.e., inability to recall an important aspect of the trauma)

C. The traumatic event is persistently reexperienced in at least one of the following ways: recurrent images, thoughts, dreams, illusions, flashback episodes, or a sense of reliving the experience; or distress on exposure to reminders of the traumatic event.

D. Marked avoidance of stimuli that arouse recollections of the trauma (e.g., thoughts, feelings, conversations, activities, places, people).

E. Marked symptoms of anxiety or increased arousal (e.g., difficulty sleeping, irritability, poor concentration, hypervigilance, exaggerated startle response, motor restlessness).

F. The disturbance causes clinically significant distress or impairment in social, occupational, or other important areas of functioning or impairs the individual's ability to pursue some necessary task, such as obtaining necessary assistance or mobilizing personal resources by telling family members about the traumatic experience.

G. The disturbance lasts for a minimum of 2 days and a maximum of 4 weeks and occurs within weeks of the traumatic event.

$\mathrm{H}$. The disturbance is not due to the direct physiological effects of a substance (e.g., a drug of abuse, a medication) or a general medical condition, is not better accounted for by Brief Psychotic Disorder, and is not merely an exacerbation of a preexisting Axis I or Axis II disorder.

Note. From Diagnostic and Statistical Manual of Mental Disorders, fourth edition, by the American Psychiatric Association, 1994, pp. 431-432.

Copyright 1994 by the American Psychiatric Association. Reprinted with permission of the author. 
arm. Second, an ASD diagnosis may predict subsequent PTSD. Hence, early diagnosis and treatment of ASD may reduce the likelihood of later chronic pathology. Third, a benefit of including ASD would be to stimulate research on acute stress reactions.

Although the inclusion of ASD in DSM-IV has, indeed, stimulated much research, the validity of the diagnosis, and especially its emphasis on dissociation, has been seriously questioned (for reviews, see Bryant \& Harvey, 2000a; Harvey \& Bryant, 2002; R.D. Marshall, Spitzer, \& Liebowitz, 1999). The emphasis on dissociative responses rests on the belief that dissociation reflects pathological cognitive avoidance that impedes emotional processing and recovery from trauma (see van der Kolk \& van der Hart, 1989). However, a conflicting view posits that dissociation during a traumatic experience may serve a protective function by attenuating the emotional impact of trauma (Horowitz, 1986; Noyes \& Kletti, 1977). Critics also worry that the ASD diagnosis amounts to inappropriate classification of a normative human response to overwhelming trauma as a medical disorder. According to this critique, the reactions embodied in the ASD criteria do not arise from underlying psychobiological dysfunction and therefore do not reflect mental illness, but rather arise from the expectable workings of evolved cognitive and emotional mechanisms for responding to trauma (see Wakefield, 1992, 1996).

The ASD diagnosis not only has these conceptual problems, but also has a weak empirical foundation, at best. Indeed, the diagnosis was included in DSM-IV without having undergone the empirical scrutiny required of other candidate diagnoses (Bryant, 2000). Even its advocates acknowledged that the predictive relation between ASD and PTSD was "based more on logical arguments than on empirical research" (Koopman,
Classen, Cardeña, \& Spiegel, 1995, p. 38). (The notion that something ought to be elevated to the status of a mental disorder because it supposedly increases risk for another disorder is an odd idea. Should high levels of cholesterol constitute a disorder because they increase risk for heart disease?) For these reasons, the diagnosis has been the subject of lively debate (e.g., Bryant \& Harvey, 2000b; R.D. Marshall et al., 1999; R.D. Marshall, Spitzer, \& Liebowitz, 2000; Spiegel, Classen, \& Cardeña, 2000).

Given that the modal outcome following trauma is recovery, can the ASD diagnosis identify persons destined to remain impaired? To date, 12 prospective studies have addressed whether the presence of ASD predicts later PTSD (Brewin et al., 1999; Bryant \& Harvey, 1998; Creamer, O’Donnell, \& Pattison, in press; Difede et al., 2002; Harvey \& Bryant, 1998b, 1999, 2000b; Holeva, Tarrier, \& Wells, 2001; Kangas, Henry, \& Bryant, in press; Murray et al., 2002; Schnyder et al., 2001; Staab, Grieger, Fullerton, \& Ursano, 1996; see Table 3). Marked methodological variability across studies likely contributes to discrepant findings regarding the relation between ASD and PTSD. For example, very low incidence rates of ASD and PTSD in certain studies may have arisen from extremely restrictive inclusion criteria, such as including only patients with very severe physical injuries (e.g., Creamer et al., in press: Schnyder et al., 2001). There is also marked variability in the types of trauma studied, ranging from motor vehicle accidents to assaults, natural disasters, and burns. As Table 3 indicates, there are two ways to evaluate the results of these prospective studies. When one looks at the proportion of people who initially displayed ASD and subsequently developed PTSD, the ASD diagnosis appears to predict PTSD reasonably well (e.g., Brewin et al., 1999; Bryant \& Harvey, 1998; Harvey \& Bryant,

Table 3. Summary of prospective studies of acute stress disorder

\begin{tabular}{|c|c|c|c|}
\hline Trauma type & Study & $\begin{array}{c}\text { Percentage of people } \\
\text { with ASD who } \\
\text { develop PTSD }\end{array}$ & $\begin{array}{c}\text { Percentage of people } \\
\text { with PTSD who } \\
\text { had ASD }\end{array}$ \\
\hline Motor vehicle accident & Harvey \& Bryant (1998b) & 78 & 39 \\
\hline Brain injury & Bryant \& Harvey (1998) & 83 & 40 \\
\hline Assault & Brewin, Andrews, Rose, \& Kirk (1999) & 83 & 57 \\
\hline Motor vehicle accident & Holeva, Tarrier, \& Wells (2001) & 72 & 59 \\
\hline Motor vehicle accident & Creamer, O'Donnell, \& Pattison (in press) & 30 & 34 \\
\hline Accidents & Schnyder, Moergeli, Klaghofer, \& Buddeberg (2001) & 34 & 10 \\
\hline Typhoon & Staab, Grieger, Fullerton, \& Ursano (1996) & 30 & 37 \\
\hline Cancer & Kangas, Henry, \& Bryant (in press) & 53 & 61 \\
\hline Motor vehicle accident & Harvey \& Bryant (1999) & 82 & 29 \\
\hline Brain injury & Harvey \& Bryant (2000b) & 80 & 72 \\
\hline Motor vehicle accident & Murray, Ehlers, \& Mayou (2002) & $77^{\mathrm{a}}$ & 34 \\
\hline Burns & Difede et al. (2002) & 87 & 78 \\
\hline
\end{tabular}

Note. $\mathrm{ASD}=$ acute stress disorder; PTSD $=$ posttraumatic stress disorder.

${ }^{a}$ This rate is based on assessments conducted 4 weeks after the trauma; the proportion of participants with ASD who developed PTSD was $32 \%$ when ASD was assessed 1 week after the trauma. 
1998b, 1999, 2000b). Across these studies, approximately three quarters of trauma survivors with ASD subsequently developed PTSD.

In contrast, the predictive ability of the ASD diagnosis is less promising when one calculates the proportion of people who eventually developed PTSD and who initially displayed ASD. This approach reveals that although some reports indicated that the majority of people with PTSD initially displayed ASD, most studies found that only a minority of people with PTSD suffered ASD within the initial month after trauma exposure. That is, the capacity of the ASD diagnosis to accurately identify most people who will eventually develop PTSD appears limited. The limitations of the ASD diagnosis as a reliable and sensitive predictor of subsequent PTSD have also been underscored by recent evidence that the ASD diagnosis may not be superior to PTSD criteria (employed within the initial month after trauma exposure) as a means of identifying people who will subsequently develop PTSD (Brewin, Andrews, \& Rose, 2003). Further, although Difede et al. (2002) found that $87 \%$ of burns survivors with ASD subsequently developed PTSD, they also reported that applying the PTSD criteria (except duration of symptoms) 2 weeks after burn injury identified the same individuals as developing PTSD.

One major reason for the variability in prospective studies of ASD and PTSD may be the timing of assessments of ASD. Although DSM-IV stipulates that ASD can be diagnosed after 2 days have elapsed since trauma exposure, it is likely that attempting a diagnostic decision this soon will increase the likelihood that a transient stress reaction will be incorrectly classified as a case of ASD. Indeed, Murray et al. (2002) found that the predictive value of the ASD diagnosis depended on when the patients were assessed. Among survivors of motor vehicle accidents, $77 \%$ of those who met ASD criteria at 4 weeks developed PTSD, compared with only $32 \%$ of those who met ASD criteria at 1 week after trauma exposure. The rapidly changing nature of stress reactions in the initial weeks following trauma exposure is underscored by evidence from studies of civilians involved in the Gulf War, in which many people who suffered immediate stress reactions in the initial days displayed marked adaptation in the following weeks (Solomon, Laor, \& McFarlane, 1996). Attempts to distinguish between transient stress reactions and harbingers of chronic disorder on the basis of symptoms expressed within days of trauma exposure will likely be very difficult.

It appears that the major reason why the ASD diagnosis fails to identify many people who eventually develop PTSD (see the right-most column in Table 3 ) is that the requirement that three dissociative symptoms be present excludes many people who nonetheless develop PTSD. For example, Harvey and Bryant (1998b) reported that $60 \%$ of trauma survivors who displayed acute reexperiencing, avoidance, and hyperarousal, but no dissociation, developed PTSD. This pattern undermines the claim that acute dissociation is a necessary harbinger of subsequent pathology.

\section{PREVENTING POSTTRAUMATIC PSYCHOPATHOLOGY}

Although many people experience acute stress-related symptoms in the wake of traumatic events, only a minority develop ASD, PTSD, or both. Most people recover from traumatic events without any professional assistance. But given that a significant minority of people exposed to trauma do develop lasting psychological problems, what sort of interventions should be offered, when should they be offered, and to whom? When considering these issues, one should be mindful of important distinctions between different kinds of interventions. Primary prevention of PTSD and other posttraumatic problems (e.g., ASD, depression, substance abuse) entails taking steps to reduce the frequency of traumatic events (e.g., restricting adolescents' access to firearms to diminish risk of school violence). These steps usually fall within the bailiwick of law and public health rather than clinical psychology and psychiatry. Secondary prevention comprises crisis intervention techniques, such as psychological debriefing, that are delivered within days of the trauma and designed to mitigate distress and prevent the emergence of posttraumatic psychopathology. Early treatment interventions are delivered soon after posttraumatic disorders have emerged, but early in the course of the disorders.

In this review, we concentrate on whether secondary prevention, especially the widely used psychological debriefing, and early treatment interventions promote recovery from posttraumatic stress. We acknowledge that survivors and communities have many needs in the aftermath of trauma, and that the prevention of persistent symptoms of psychological distress is only one of them. It is, however, beyond our scope here to review the many different targets of crisis intervention and their effectiveness.

\section{PSYCHOLOGICAL DEBRIEFING}

Psychological debriefing has its roots in World War I (Litz, Gray, Bryant, \& Adler, 2002). Following a major battle, commanders would meet with their men to debrief them. The objective was to boost morale by having combatants share stories about what had happened during the engagement. This historical group debriefing method was also used by American troops during World War II and continues to be used by the Israeli army today (Shalev, Peri, Rogel-Fuchs, Ursano, \& Marlowe, 1998).

Drawing parallels between the stress of combat and the stress of emergency medical service, Mitchell (1983) reasoned that a similar approach might diminish stress reactions among firefighters, police officers, emergency medical technicians, and other people exposed to what he referred to as "critical incidents" (i.e., traumatic events). A former firefighter and paramedic, Mitchell obtained a Ph.D. in human development and developed the most widely used method of psychological de- 
briefing: Critical Incident Stress Debriefing (CISD). In his seminal article, Mitchell (1983) emphasized that too many people believe that firefighters, police, and other emergency service personnel are emotionally impervious to trauma. Contrary to the John Wayne stereotype, he said, "Rescuers are vulnerable human beings who have all the normal physical and psychological responses to the horror of human suffering" (Mitchell, 1983, p. 36). That is, helping the primary victims of trauma might constitute a major stressor for the helpers themselves. Accordingly, Mitchell asserted that the mental health of emergency personnel who respond to a critical event is best served when they participate in a structured session enabling them to talk about the event and ventilate their emotions, especially in the company of peers who have experienced the same incident.

CISD is designed to mitigate the adverse psychological consequences of traumatic events by attenuating the intensity of acute symptoms of stress, thereby reducing the risk of subsequent psychiatric problems. A single debriefing session "will generally alleviate the acute stress responses which appear at the scene and immediately afterwards and will eliminate, or at least inhibit, delayed stress reactions" (Mitchell, 1983, p. 36). Originally developed for emergency service personnel, CISD is now deemed helpful for "primary victims" (i.e., the people directly exposed to trauma; Everly \& Mitchell, 1999, p. 85) as well. (However, in 2002, Mitchell continued to dismiss studies showing psychological debriefing had no beneficial impact if they involved primary victims.) Debriefings now occur in businesses, schools, hospitals, and the military (Everly \& Mitchell, 1999, pp. 84-85). There are different versions of psychological debriefing (Raphael \& Wilson, 2000), but "Mitchell's CISD model of psychological debriefing is generally recognized as the most widely used in the world and is used across the greatest diversity of settings and operational applications" (Everly \& Mitchell, 1999, p. 84).

Initially, Mitchell (1983) described a debriefing session as "either an individual or group meeting between the rescue worker and a caring individual (facilitator) who is able to help the person talk about his feelings and reactions to the critical incident" (p. 37). However, since the late 1980s, Mitchell has argued that CISD should be delivered only to groups of individuals who have been exposed to a critical incident, not to single individuals. That is, although originally deemed suitable for either individuals or groups, CISD is now recommended only for groups.

A CISD session lasts between 3 and $4 \mathrm{hr}$ and is conducted between 2 and 10 days after a critical incident, except in mass disasters, in which case it occurs about 3 to 4 weeks later (Everly \& Mitchell, 1999, p. 18). According to its advocates, debriefing works because it is delivered soon after the trauma, because it provides psychosocial support and an opportunity for expressing emotions and thoughts about the trauma, and because it provides tips on coping and education about stress and its management.
A debriefing session has seven phases. In the introduction phase, the debriefing facilitator explains the procedure to the participants, answering any questions they might have. He or she emphasizes that debriefing is not psychotherapy; it is a method for reducing normal stress reactions triggered by a horrific event.

The facilitator then initiates the fact phase by asking each participant, in turn, to describe what happened during the critical incident. He or she might say, "Tell me who you are, what your role in the incident was, and just what you saw and/or heard take place" (Everly \& Mitchell, 1999, p. 86). The purpose is to enable each person to describe the traumatic incident from his or her perspective. "Each person takes a turn adding in the details to make the whole incident come to life again in the CISD room" (Mitchell, 1983, p. 38). However, the facilitator reassures participants that they can remain silent if they feel uncomfortable speaking in the group. The option of silence also applies to subsequent phases of the debriefing.

Next, the facilitator shifts to the thought phase by allowing each participant to describe his or her cognitive reactions to the traumatic event. The facilitator might say, "Now, I'd like you to tell us what your first thoughts were in response to the crisis" (Everly \& Mitchell, 1999, p. 86). The purpose of this phase is to move closer to the expression of emotion.

The facilitator then moves to the reaction phase-the one designed to foster emotional processing of the trauma by having participants experience catharsis through expressing their feelings about the event. The facilitator might begin by asking, "What was the worst part of the incident for you personally?" (Everly \& Mitchell, 1999, p. 86). The facilitator may ask how each person felt then and also how each person is feeling during the debriefing itself. As Mitchell (1983) has emphasized, "Everyone has feelings which need to be shared and accepted. The main rule is-no one criticizes another; all listen to what was, or is, going on inside each other" (p. 38).

Advancing to the symptom phase, the facilitator asks, "What physical or psychological symptoms have you noticed, if any, as a result of this incident?" (Everly \& Mitchell, 1999, p. 87). The purpose of this phase is to identify stress reactions that members wish to share, and to begin the transition from the affective realm back to the cognitive one.

In the teaching phase, the facilitator tries to show that the stress reactions members have been experiencing are normal and not necessarily a medical problem, by stating, for example, "We've heard numerous symptoms that are being experienced, let me explain their nature and give you some suggestions on how to reduce their negative impact" (Everly \& Mitchell, 1999, p. 87). In addition to providing stress-management tips, he endeavors to convince participants that their reactions do not signify psychopathology.

Finally, in the reentry phase, the facilitator aims to achieve closure to the traumatic event. He or she summarizes what has been covered in the session, answers any questions that have arisen, and assesses whether any members may need follow-up or referral for additional services. 
Although Mitchell (1983) originally asserted that "the formal CISD should be mandatory for all personnel involved in the scene" (p. 38), he has since acknowledged that compelling people to undergo debriefing raises "intriguing" issues (Everly $\&$ Mitchell, 1999, p. 93). On the one hand, allowing participation to be voluntary "runs the risk of under utilization based upon the stigma of needing help" (Everly \& Mitchell, 1999, p. 93). On the other hand, mandatory debriefing "raises issues of coercion, legal liability, and informed consent" (Everly \& Mitchell, 1999, p. 93). As a possible solution to this dilemma, Mitchell and Everly have suggested providing a mandatory general information session for everyone involved in the critical incident and then following up with voluntary formal debriefings. In any event, they recommend that anyone exposed to a critical incident be offered debriefing, regardless of whether the person is experiencing any stress symptoms.

In addition to mitigating distress and preventing posttraumatic problems, Mitchell has argued, these interventions may reduce sick days taken by stressed employees (Everly \& Mitchell, 1999, pp. 131-135). "Not only do [these] services make sense from a humanitarian perspective, they make sense from a business perspective, as well" (Everly \& Mitchell, 1999, p. 135). According to Everly and Mitchell (1999, p. 135), a business's failure to implement some such psychological service in the immediate wake of a critical incident may constitute negligence, thereby increasing the risk of legal liability to stressed employees who may file suit. To avoid the threat of litigation for failing to meet the standard of care, some police departments in the United Kingdom have now made debriefing compulsory for people exposed to critical incidents; banks in the United Kingdom and Australia have also made debriefing compulsory for employees exposed to critical incidents in the workplace (Rose, Bisson, \& Wessely, 2001). Everly and Mitchell (1999, p. 135) predicted that their approach might emerge as "the standard of care" for intervention in the wake of crisis and trauma. Indeed, between 30,000 and 50,000 individuals are trained each year by their organization, the International Critical Incident Stress Foundation, Inc. (ICISF). ${ }^{3}$

In the 1990s, Everly and Mitchell (1999) expanded the range of crisis intervention services offered by ICISF. The proprietary term for the entire set of techniques is Critical Incident Stress Management (CISM). The overarching purpose of CISM is "to reduce the incidence, duration, and severity of, or

3. Kadet (2002) reported that 40,000 people are trained in Mitchell and Everly's methods each year. On March 2, 2002, she contacted Don Howell, the executive director of the ICISF, to ask him how many people are trained by ICISF each year. According to her notes (A. Kadet, personal communication, April 2, 2003), Howell said, "We do 30 to 50 thousand people a year; that's a conservative number. We've been at that pace for the past 4 or 5 years." When the fact checker for Smart Money magazine recontacted him to double-check the figures, he put the figure at 40,000 individuals trained annually. In a training workshop given by Mitchell in Columbia, Maryland, on May 30, 2002, he said his organization trains 30,000 people per year (S. Satel, personal communication, April 5, 2003). impairment from, traumatic stress" (Everly \& Mitchell, 1999, p. 72). Hence, CISM is not a technique or method per se; it is a framework or strategy comprising a set of tactics, each designed to meet a distinct crisis intervention goal. Grouped under the CISM rubric are the following methods, in addition to CISD (Everly \& Mitchell, 1999, pp. 71-92):

- Pre-incident preparedness training refers to educating individuals in high-risk occupations (e.g., firefighters, emergency medical technicians) about the kinds of stressors they are likely to encounter on the job, about common stress reactions, and about stress-management techniques.

- One-on-one individual crisis support refers to attempts to mitigate acute stress reactions, often at the scene of the trauma. The counselor attempts to provide psychological distance between the scene and the person in distress by having the person take a walk, get a cup of coffee, and so forth. As Everly and Mitchell (1999) pointed out, "In actuality, most crisis response interventions will be done individually, that is, one-on-one, rather than in groups" (p. 76).

- Demobilization refers to providing food, rest, and information about coping with stress reactions to large groups of disaster personnel as they rotate off duty. This method includes group informational briefing, which refers to providing facts about a critical incident (e.g., a student's suicide) to a large group of individuals indirectly affected, as well as providing information about common psychological dynamics (e.g., grief, anger) and about how to access psychological services. This tactic usually applies to schools and businesses affected by a critical incident.

- Defusing refers to a small-group intervention that usually takes place within $12 \mathrm{hr}$ of the traumatic event. It involves having participants explore and discuss the incident and their emotional reactions to it. Group leaders teach coping skills and tell participants that stress reactions are normal and expected, and do not necessarily signify mental illness. Otherwise similar to CISD, defusing can be repeated.

- Family support refers to debriefing family members of the persons involved in the crisis (e.g., spouses of individuals in the military).

- Referral mechanisms concern procedures for referring individuals for psychiatric or psychological services, legal services, career counseling, and so forth.

\section{Does Psychological Debriefing Work?}

Most people who receive debriefing endorse it as helpful (e.g., Carlier, Voerman, \& Gersons, 2000; Small, Lumley, Donohue, Potter, \& Waldenstrom, 2000). But this does not mean that it prevents posttraumatic mental disorders. These reports that the method is helpful may reflect little more than polite expressions of gratitude for attention received. Given that only some trauma-exposed people will develop PTSD-and many recover on their own - the efficacy of debriefing can be gauged only by comparing the outcomes for individuals who 
did and did not receive this intervention. If a certain crisis intervention method fails to reduce symptoms and prevent disorder, then it should be discontinued in favor of developing something that actually does work.

Some scholars are convinced of debriefing's efficacy. Mitchell and Everly (2001) argued, "The experiences of 700 CISM teams in more than 40,000 debriefings cannot be ignored. This is especially so when the overwhelming majority of the reports of debriefing services are extremely positive" (p. 295). Mitchell and Everly (2001) claim that "numerous studies have already been published with very positive results" (p. 295), and that research on their methods "proves their clinical effectiveness far beyond reasonable doubt" (Mitchell \& Everly, 2001, p. 84; see also Everly, Flannery, \& Eyler, 2002).

Other scholars, who have published meta-analyses, have drawn dramatically different conclusions. Rose et al. (2001) concluded that "there is no current evidence that psychological debriefing is a useful treatment for the prevention of post traumatic stress disorder after traumatic incidents. Compulsory debriefing of victims of trauma should cease" (pp. 1-2). Another meta-analysis revealed that trauma-exposed individuals who had not received CISD experienced reductions in PTSD symptoms, whereas those who had received CISD did not (van Emmerik, Kamphuis, Hulsbosch, \& Emmelkamp, 2002).

Rose et al. (2001) confined their meta-analysis to randomized controlled trials (RCTs) of psychological debriefing. The RCT is the standard method for testing the efficacy of any intervention, psychological or pharmacological. It requires that subjects be randomly assigned to receive either the intervention or no treatment. Reliable and valid measures of symptoms are administered to both groups both before and after the intervention is delivered to the treatment group, and readministered at follow-up, usually 6 months to several years later. If the intervention works as predicted, individuals who received the intervention should have better outcomes than those who did not. A failure to include a no-treatment (or assessment-only) control group makes it impossible to distinguish between improvements attributable to the intervention and those attributable to natural processes occurring with the passage of time. In the following review, we discuss all the RCTs on psychological debriefing that were included in the meta-analyses of Rose et al. and van Emmerik et al. (2002) and were published in journals; we also discuss some non-RCTs.

For several reasons, using the RCT model to test the efficacy of crisis intervention methods is more complicated than using it to test the efficacy of psychotherapy or pharmacotherapy. First, investigators may object to randomly assigning trauma-exposed individuals to a no-treatment control condition. Depriving them of a potentially helpful treatment seems to raise ethical issues. Of course, this objection presupposes that the intervention is, indeed, effective. If an intervention is not known to work, there is no ethical problem in withholding it. Moreover, some interventions may impede natural recovery, making it essential that RCTs test the efficacy of all interventions. Second, unless psychological measures are already available (e.g., predeployment measures of psychological functioning among soldiers about to embark on a military mission), it is difficult to get pretrauma assessments. Third, debriefing is often administered following man-made or natural disasters, unexpected events that produce much chaos. Unless somehow prepared in advance to conduct such studies, researchers are often unable to devise a proposal, secure ethical (human subjects) approval, and launch a study in a timely fashion.

Everly and Mitchell (1999, pp. 109-110) believe that the merits of RCTs have been overstated. More specifically, they argue that random assignment to debriefing and control groups is extremely difficult, especially in a crisis intervention context, and may undermine the probative import of the findings. Attempts to increase internal validity (experimental control) may wind up sacrificing external validity (generalizability to the real world of crisis intervention). That is, in real-world crisis situations, individuals are not randomly assigned to no-treatment control conditions preceded and followed by clinical assessments, whereas these features are integral to RCTs.

How is it possible for scholars to arrive at dramatically different conclusions about the value of psychological debriefing? Close reading of the publications of the critics and the advocates of debriefing reveals that much of the time, they are relying on different sources of evidence to arrive at their conclusions. Debriefing advocates cite allegedly positive findings that the critics regard as suffering from fatal methodological flaws (e.g., failure of randomized assignment, absence of control groups). Debriefing critics cite other studies that advocates claim fail to provide proper tests of the method (e.g., studies testing one-on-one debriefings rather than group debriefings). Therefore, to clarify this contentious issue, we first review the data debriefing advocates adduce in favor of the method. We then review the data debriefing critics adduce against the method. Finally, we review and evaluate the replies of debriefing advocates to the "negative" studies.

\section{Studies Adduced in Support of Debriefing}

A few published, peer-reviewed studies are included among the research adduced as confirming the efficacy of debriefing (Everly \& Mitchell, 1999, pp. 107-129; Mitchell, 2002). Conducting methodologically sound research in this area is very challenging. Nevertheless, one's confidence in the efficacy of debriefing (or other interventions) is enhanced when researchers assign participants randomly to the treatment and no-treatment control conditions, deliver a standardized intervention with reasonable fidelity to the protocol, use reliable and valid measures of psychological distress, and conduct subsequent assessments to determine whether debriefed individuals are doing better than nondebriefed individuals at follow-up. 
In an early pre-CISD study cited as relevant to CISM by Everly and Mitchell (1999), Bordow and Porritt (1979) randomly assigned 70 male patients who had been hospitalized following road traffic accidents to a one-on-one crisis intervention condition or to no intervention. The intervention comprised providing practical assistance (e.g., obtaining financial assistance from welfare agencies), exploring emotional reactions, and encouraging family members to be supportive of the patient. During assessments 3 to 4 months later, the crisis intervention group reported significantly fewer psychiatric symptoms than did the untreated group, whose members improved very little.

In another study cited in support of crisis intervention (Mitchell, 2002), Bunn and Clarke (1979) randomly assigned 30 individuals, who had accompanied a seriously injured relative to the hospital, to receive a 20-min crisis intervention counseling session or no treatment. Although this study was done before Mitchell (1983) developed CISD, the intervention did contain certain elements similar to those of CISD, such as providing information and empathic support, and encouraging participants to express feelings about the crisis. The researchers audiotaped two 5-min speech samples-one before and one after the 20-min intervention-provided by each of the individuals who accompanied their injured relatives to the hospital. (Thus, the participants were not the injured persons, but rather the individuals who accompanied them to the hospital.) The speech samples of the accompanying individuals were rated and scored for expressions of "anxiety." These scores indicated that counseled individuals had a significant decrease in anxiety, whereas participants who received no treatment did not. The brevity of the follow-up period-20 min! — and the unvalidated anxiety measures used make these findings difficult to interpret.

Wee, Mills, and Koehler (1999) published the most encouraging, albeit flawed, study on CISD. They asked emergency medical service personnel, who either had or had not received CISD after having worked during the 1992 Los Angeles riot, to complete a PTSD questionnaire. Logistical constraints prevented the 23 nondebriefed individuals from receiving the otherwise-mandatory CISD. Most participants (72.9\%) reported having been attacked by the rioters, so they were primary victims of trauma. Three months after the civil disturbance, the 42 debriefed participants reported significantly fewer PTSD symptoms than did the nondebriefed participants. Unfortunately, the absence of random assignment and assessment of symptoms before the intervention diminish the probative import of the study.

Other studies adduced by Everly and Mitchell (1999, pp. 107-129; Mitchell, 2002, p. 21) in support of debriefing fail to provide much convincing evidence in favor of the method. For example, Amir, Weil, Kaplan, Tocker, and Witztum (1998) studied 15 Israeli women who had survived a terrorist attack on a bus on which they were riding. These women received a group debriefing 2 days after the attack, followed by six group psychotherapy sessions occurring during the following 2 months. Self-reported PTSD symptoms declined between 2 days posttrauma and 2 months posttrauma, but the lack of a no-treatment control group renders any symptomatic change impossible to interpret. Moreover, the outcome measures conflate the effects of the debriefing and the six group psychotherapy sessions. In any event, $27 \%$ of the women still met the criteria for a diagnosis of PTSD at 6 months, prompting the authors to state that "it can safely be concluded that the intervention had little if any effect" (Amir et al., 1998, p. 241).

Campfield and Hills (2001) randomly assigned robbery victims to receive either an immediate debriefing (within $10 \mathrm{hr}$ of the crime; $n=36$ ) or a delayed debriefing (more than $48 \mathrm{hr}$ after the crime; $n=41$ ). Mitchell's (1983) method was followed, and individuals were debriefed either individually or in groups usually ranging from 2 to 4 victims. The immediate-intervention group reported significantly fewer PTSD symptoms at 2 days, 4 days, and 2 weeks postintervention, whereas the delayed-intervention group reported no decline in symptoms during this period. The authors and Mitchell (2002) interpreted these findings as support for immediate debriefing.

Unfortunately, Campfield and Hills's (2001) findings are difficult to interpret because of the short follow-up period and absence of a no-treatment control group. That is, it is entirely possible that a group of robbery victims who received no debriefing would have shown even faster recovery than those receiving either of the debriefing interventions. Furthermore, the study violates three of Mitchell's (2002) criteria for a proper assessment of debriefing. First, in discussing published studies showing debriefing has no effect, Mitchell $(2002$, p. 18) criticized the researchers for committing an "egregious" error by using change in PTSD symptoms as a dependent variable to gauge the impact of debriefing. In contrast, he praised Campfield and Hills's study for showing a significant decline in PTSD symptoms (p. 24). (Moreover, a decline in symptoms during the initial 2 weeks is likely attributable to natural recovery anyway.) Second, whereas Mitchell emphasizes that standard CISD is designed for emergency service personnel, not primary victims of trauma, the participants were all primary victims of robberies. Third, although Mitchell (2002) has stated that CISD should be delivered only to groups, most participants in this study were treated either individually or as pairs (i.e., average "group" size was 2.5 people per debriefing). In summary, because Campfield and Hills's study violated several of Mitchell's criteria for an adequate test of debriefing, it is puzzling that he affirmed its probative value.

Yule (1992) conducted a study of British schoolchildren who survived the sinking of a cruise shop. Ten days after the shipwreck, clinicians had delivered a single debriefing session to two small groups of the children (total $n=24$ ). Children at another school whose administration declined a debriefing $(n=15)$ served as a contrast group. There was no random assignment to groups, and there was no predebriefing assessment. 
Five to 9 months after the disaster, the children completed selfreport measures of PTSD, anxiety, fears, and depression. The children who had received the debriefing reported significantly fewer intrusive symptoms and fewer fears unrelated to the disaster than did the control group; there was also a nonsignificant trend suggesting that the debriefing group had fewer avoidance symptoms and fears related to the shipwreck. Unfortunately, the lack of random assignment and preintervention assessment complicate interpretation of the data. Moreover, Yule described the single session as "a problem-solving approach, based on cognitive behavioural methods" (p. 203) rather than as CISD.

Deahl et al. (2000) studied 106 British soldiers assigned to 6 months of peacekeeping duty in Bosnia. Prior to their deployment to Bosnia, all the soldiers had received an Operational Stress Training Package that included information about stress and its management. Upon their return from Bosnia, Deahl et al. asked the commanding officers to allocate the soldiers into two groups, and they "did so according to individual availability and commitment to other duties" (Deahl et al., 2000, p. 79). One group ( $n=54$ ) received a single session of debriefing, based on Mitchell's (1983) model and conducted by experienced debriefers, whereas the second group $(n=52)$ received no intervention. The debriefing session lasted about $2 \mathrm{hr}$, and was conducted in groups of 8 to 10 soldiers. The debriefing and no-treatment groups were both assessed prior to the first group's debriefing and again 3, 6, and 12 months later.

Although a significantly larger proportion of the control group $(25 \%)$ than of the debriefed group $(7.4 \%)$ retrospectively reported experiencing "intense distress" (Deahl et al., 2000, p. 80) while they were in Bosnia, the control group experienced a significant decrease in PTSD symptoms (assessed by questionnaire) by the 6-month assessment, whereas the debriefed group did not. PTSD symptoms assessed by clinical interview did not differ between the two groups at any time point. Indeed, only 2 soldiers in the control group and only 1 soldier in the debriefing group met the criteria for a diagnosis of PTSD at any time since returning from Bosnia. At the 6-month and 12-month assessments, the debriefed group reported significantly fewer symptoms than the control group on a questionnaire tapping anxiety and depressive symptoms. Both groups scored high on a self-report measure of alcohol abuse, but only subjects in the debriefed group evinced a significant reduction in these symptoms. The very low level of problems among both groups led Deahl et al. (2000) to conclude that high rates of psychiatric illness are not inevitable consequences of military conflict. Contrary to Mitchell's (2002) guidelines, the participants in this study were direct recipients of trauma (soldiers) rather than secondary recipients (i.e., emergency service personnel), and the authors themselves described their study as "not a true RCT of debriefing because selection of the sample was restricted, the method of randomization for debriefing was less than ideal and the low level of PTSD symptoms at the outset meant that there was little scope for reduction" (Deahl et al., 2000, p. 83). Mitchell (2002) interpreted this study by Deahl et al. as support for debriefing, emphasizing, for example, the significant reduction in alcohol consumption in the debriefed group.

Chemtob, Tomas, Law, and Cremniter (1997) reported that a single group debriefing session significantly reduced selfreported PTSD symptoms of people exposed to a hurricane that had struck one of the Hawaiian islands. This study did not include a nondebriefed control group. Moreover, the debriefing was delivered 6 months after the trauma. Because debriefing is specifically deemed an early intervention, it should be delivered within days after the trauma, not half a year later. Chemtob et al. acknowledged that their procedure diverged from the standard approach, noting "the length of time between the event and the intervention" (p. 417). The treatment, in effect, was a single psychotherapy session rather than a preventive crisis intervention.

In an even more striking departure from protocol, Busuttil et al. (1995) reported data on 34 individuals who received multiple debriefings within a 12-day residential treatment program. All subjects already had PTSD, 19 of them for 2 to 31 years! One year after treatment, $85 \%$ no longer had PTSD. There was no control group. Although debriefing was delivered in groups, the traumatic events suffered by members of the same debriefing group differed substantially, and the participants were primary, not secondary, victims (e.g., survivors of the Falkland Islands War and car accidents, former hostages). Finally, Busuttil et al. characterized their program as "group psychotherapy" (p. 495), not crisis intervention.

Stallard and Law (1993) reported data on 7 adolescents who had escaped from a minibus accident with only minor injuries. Six months later, the youngsters completed PTSD, depression, and anxiety measures prior to receiving two group debriefing sessions. Three months after the intervention, scores on all scales indicated improvement. However, there were too few participants for the study design to include random assignment to a no-treatment control group, making the symptom reductions difficult to interpret. Also, because the debriefing took place months after the trauma, this study is not relevant to crisis intervention. Nevertheless, Everly, Boyle, and Lating (1999) included it in their meta-analysis as support for psychological debriefing.

Nurmi (1999) conducted a single debriefing with small groups of rescue workers 3 to 7 days after the sinking of a ship near Finland. There was some evidence that scores on selfreport (including PTSD) measures were lower among occupational groups whose members were debriefed (e.g., firefighters) than among occupational groups whose members were not debriefed (e.g., female nurses). But lack of randomization, lack of predebriefing measures of symptoms, and the presence of gender confounds (e.g., certain nondebriefed occupational groups, such as nurses, had only female participants) render the findings nearly uninterpretable. 
Studying groups of British bank employees who had been present during robberies, Richards (2001) collected data on PTSD symptoms 3 days, 1 month, and 3 to 12 months posttrauma. The banks had been conducting single-session CISDs following robberies, but they later incorporated CISD into a comprehensive employee program (i.e., a CISM framework) involving, for example, educational programs on robberies and stress. Richards compared longitudinal symptom reductions during the CISD-only era with those occurring after CISM had been instituted, noting trends indicating outcomes at long-term follow-up were more favorable after the full-fledged CISM program was in effect. It is very difficult to make confident inferences from such a design given the consecutive sequence (CISD followed by CISM), lack of random assignment to groups, and lack of a control group. And it is impossible to attribute the reductions over time to either CISD or to CISM; most people who witness traumatic events, such as robberies, will improve regardless of any intervention.

Leeman-Conley (1990) described a crisis intervention program established in Australia to provide counseling and support for bank employees who are present during bank robberies. After a holdup, each employee is seen individually by a counselor, and then participates in a group session the next day. Employees took $60 \%$ fewer sick days off work during the first 2 years after the program's implementation than they had prior to program implementation, and associated worker compensation costs declined $66 \%$ as well. Unfortunately, there was no control group, making it impossible to attribute declines in sick days and compensation payments to the program. Moreover, Leeman-Conley reported no data on any psychological symptoms (e.g., anxiety, PTSD). Although she did not cite the work of Mitchell and Everly or mention CISM, Mitchell (2002) nevertheless described this program for direct victims as a "CISM program" (p. 37).

Using Mitchell's (1983) CISD intervention, Bohl (1995) reported that firefighters $(n=30)$ who had received debriefings scored significantly lower on "psychological measures" ( $p$. 126) of depression, anger, anxiety, and "long-term stress symptoms" (p. 126) than did firefighters $(n=35)$ who had not been debriefed. Although Bohl said that she administered "objective tests" (p. 125) 3 months posttrauma, she did not say what those tests were (e.g., questionnaires?). Hers was not a randomized trial; rather, she compared responses of firefighters whose firehouse debriefed its employees with those of firefighters from a firehouse where debriefings did not occur. Moreover, it is unclear whether firefighters were debriefed in groups or oneon-one.

Jenkins (1996) administered questionnaires to 36 emergency medical workers 8 to 10 days after they had worked at the scene of a mass shooting and again about 1 month later. Shortly after the incident, $52 \%$ of the workers attended at least one CISD session, but participants were not randomly assigned to receive debriefing or not. Unfortunately, Jenkins merely cor- related self-report symptoms with other measures (e.g., of social support). She did not compare psychological outcomes in debriefed versus nondebriefed participants. Nevertheless, she concluded that the study indicates "the apparent usefulness of CISD for reducing symptoms of depression and anxiety over the month after the incident" (p. 488).

Finally, debriefing advocates (Everly \& Mitchell, 1999; Mitchell, 2002) have cited Flannery's Assaulted Staff Action Program (ASAP) as support for the efficacy of CISM (Flannery, 1999, 2001; Flannery, Fulton, Tausch, \& DeLoffi, 1991; Flannery, Hanson, Penk, Flannery, \& Gallagher, 1995; Flannery et al., 1998; Flannery, Penk, \& Corrigan, 1999; Flannery, Stone, Rego, \& Walker, 2001). Concerned that staff working on psychiatric units are at risk for developing PTSD symptoms after being attacked by mental patients, Flannery instituted a CISM-like program at an inpatient facility in Massachusetts (Flannery et al., 1991). Whenever a staff member is assaulted by a patient, a trained ASAP clinician immediately conducts a one-on-one debriefing with the staff victim. The debriefer assesses the victim's sense of emotional control, social supports, and ability to make sense out of the incident, and then contacts the victim again 3 and 10 days later. If further action is warranted, the victim is referred to a support group comprising staff members who are trying to cope with having been attacked by patients. Referrals for private counseling and family counseling are provided as needed. Occasionally, the assault is so severe that a group debriefing for all ward staff occurs.

Flannery has yet to publish any questionnaire or interview data regarding ASAP's capacity to attenuate assault-related stress symptoms, nor has he conducted an RCT of ASAP's efficacy. Interestingly, in six settings (four residential programs, two community mental health centers), Flannery (2001) has documented a significant reduction in the frequency of assaults on staff following the implementation of ASAP. This decline ranged from $25 \%$ to $62 \%$. Flannery acknowledges that these findings are uncontrolled - another variable correlated with the implementation of ASAP might explain the sudden drop in violence against staff. However, ASAP may itself have altered staff behavior, which, in turn, may have inadvertently reduced the likelihood of patients becoming violent.

In summary, the studies we have just described constitute the evidence adduced in support of psychological debriefing (Everly \& Mitchell, 1999, pp. 107-129). Because of their methodological limitations, these studies fail to provide a convincing case for the efficacy of debriefing to mitigate distress and prevent posttraumatic psychopathology.

\section{Studies Adduced as Showing Null or Adverse Effects of Psychological Debriefing}

Critics of debriefing have cited studies showing that debriefed participants fared no better-or even worse-than their 
nondebriefed counterparts on measures of posttraumatic symptoms. In their discussion of the evidence, critics have concentrated on RCTs (e.g., Rose et al., 2001). ${ }^{4}$

Following Mitchell's (1983) model of CISD, Rose, Brewin, Andrews, and Kirk (1999) randomly assigned 157 adult crime victims (118 male) to either psychological debriefing, an educational intervention, or assessment only. All had been victimized during the previous month; $94 \%$ had been physically assaulted, and the others had been victims of attempted or completed sexual assault, physical assault, or robbery. One third had suffered severe injuries (e.g., broken bones). The number of participants, however, was only a fraction of those who might have qualified. That is, Rose et al. contacted 2,161 crime victims who potentially qualified for inclusion, but only 243 replied, and of those who replied, only 157 turned out to be eligible for the study.

The debriefing lasted about $1 \mathrm{hr}$, and was delivered in an individual format. Subjects were urged to describe their traumatic experience in detail, including the facts of the crime and their thoughts and feelings during it. They were encouraged to express negative emotions, such as guilt, shame, and fear, that might otherwise have gone unexpressed. In addition, they re-

4. In their review, Rose et al. (2001) included not only studies on debriefing of individuals exposed to trauma, but also studies on debriefing of individuals who experienced other kinds of misfortunes. For example, Lee, Slade, and Lygo (1996) randomly assigned women who had recently had a miscarriage to either debriefing or no treatment. Two days after the miscarriage, the women were sent questionnaires assessing symptoms of anxiety, depression, and PTSD, as well as reactions to miscarriage. The women in the debriefing group received a 1-hr session in their homes about 2 weeks after the miscarriage. The female psychologist who did the debriefing used a protocol based on Mitchell's (1983) method. In both groups, PTSD symptoms were initially elevated, but dropped sharply by the 4-month assessment. Depression scores were not elevated at either assessment, whereas anxiety scores were elevated at both time points. Although debriefing had no effect on emotional adjustment, debriefed subjects reported it to be helpful.

Although a routine, uncomplicated birthing does not qualify as a traumatic stressor, some researchers have tested whether debriefing new mothers might reduce the incidence of postpartum maladjustment. Lavender and Walkinshaw (1998) randomly assigned women who had just given birth to either a session of debriefing $(n=56)$ or no intervention $(n=58)$. The women had experienced a normal labor and delivery. The debriefing session lasted between 30 and $120 \mathrm{~min}$, and consisted of the mother discussing the labor and delivery, getting any questions answered, and exploring her feelings about the experience. Three weeks later, debriefed mothers were significantly less likely than nondebriefed mothers to score high on a self-report measure of anxiety and depressive symptoms.

Another research group randomly assigned 1,041 women who had just given birth via an operative method (caesarian, forceps, or vacuum extraction) to either debriefing or usual postpartum care (Small et al., 2000). At 6 months postpartum, the proportion of debriefed women who were depressed $(17 \%)$ was nonsignificantly higher than the proportion of nondebriefed women who were depressed $(14 \%)$. Scores on seven of eight scales measuring general health status indicated that the debriefed women were doing worse than the nondebriefed women, although the difference was significant on only one scale (emotional role functioning). Despite the failure of debriefing to reduce postpartum depression, $51 \%$ of the debriefed subjects endorsed the debriefing session as "helpful," and another $43 \%$ rated it as "very helpful." ceived information about common reactions to traumatic events and where to get further psychological help, if necessary. All debriefing sessions were reviewed by the research team to ensure that protocol was being followed. Subjects randomly assigned to the 30-min educational intervention received only information about common reactions, whereas those in the assessment-only condition received only the assessment measures.

Assessments occurred before the intervention, and again 6 and 11 months later. At the 6-month assessment, rates of PTSD were $26 \%, 23 \%$, and $11 \%$ in the assessment-only, debriefing, and educational groups, respectively. Although the rate of PTSD was nearly twice as high in the group that received debriefing as in the group that received only education, there were no statistically significant differences among the groups in rates of PTSD. By the 11-month assessment, rates of PTSD had dropped so low in all groups that statistical analysis was pointless. All groups exhibited marked and significant improvement over time on questionnaire measures of both PTSD and depression. But the groups did not differ significantly in their improvement on these measures. The authors concluded, "No evidence was found to support the efficacy of brief, onesession interventions for preventing post-traumatic symptoms in individual victims of violent crime" (Rose et al., 1999, p. 793).

Conlon, Fahy, and Conroy (1999) randomly assigned survivors of motor vehicle accidents to either psychological debriefing $(n=18)$ or an assessment-only control group $(n=22)$. None of the subjects required hospitalization, and the 30-min debriefing session immediately followed the baseline assessment, occurring an average of 7 days after the accident. The debriefing protocol included information on common responses to traumatic events, and it encouraged subjects to express the thoughts and feelings they had experienced during the trauma. Advice on coping strategies and seeking further help was provided. Both groups improved markedly on both self-report and clinician-rated measures of PTSD symptoms, but there were no significant differences between the groups 3 months after the intervention. Conlon et al. concluded that they failed "to show any prophylactic benefit of PD [psychological debriefing] in trauma victims" (p. 43).

Debriefing advocates often emphasize that CISD is not a "one-off, stand-alone" method, meaning that it is not intended to be administered in a single session. Therefore, to test whether more extensive intervention might help, Carlier et al. (2000) administered three successive debriefing sessions, based on Mitchell's (1983) method, delivered $24 \mathrm{hr}, 1$ month, and 3 months posttrauma. In fact, regulations in The Netherlands, where this study was conducted, require that three debriefing sessions be offered to any police officer who has been exposed to a critical incident. The research team formed an "external control group" comprising 75 police officers who had been exposed to trauma prior to the introduction of debriefing. The "internal control group" comprised 82 police officers who 
had refused debriefing either because they regarded the incident as too trivial or because they lacked the time to participate. These two groups were compared with a debriefed group of 82 officers. The sessions were delivered one-on-one by a trained peer debriefer.

Ninety-eight percent of debriefed participants expressed satisfaction with the first two sessions, and the remaining $2 \%$ reported some satisfaction. However, ratings of satisfaction were unrelated to participants' number of psychological symptoms, number of sick days off work, or resumption of regular duties (Carlier et al., 2000).

Carlier et al. (2000) assessed participants shortly before the start of debriefing (i.e., pretest), shortly after the first debriefing session (i.e., $24 \mathrm{hr}$ posttrauma), 1 week posttrauma, and 6 months posttrauma (i.e., after the second and third debriefing sessions). One week after the trauma, debriefed participants reported significantly more PTSD symptoms than did nondebriefed participants. There were no differences among the groups at the 24-hr and 6-month assessments. In fact, rates of PTSD symptoms were very low across all groups.

Two studies suggest that debriefing may impede natural recovery from trauma. Bisson, Jenkins, Alexander, and Bannister (1997) randomly assigned hospitalized burn victims to either debriefing ( $n=57$ ) or an assessment-only control condition $(n=46)$. The debriefing session occurred between 2 and 19 days after the accident, and it lasted between 30 and $120 \mathrm{~min}$. Mitchell's (1983) protocol was used. The partners (usually a spouse) of 16 of the 57 debriefed individuals attended the debriefing sessions, whereas the remaining subjects were debriefed alone. Bisson et al. compared the groups on 10 measures of trauma severity (e.g., percentage of body burned, pain). The debriefing group scored significantly $(p<.05)$ higher than the control group on only 1 measure (whether other people were involved in the accident), although nonsignificant trends for 4 other severity measures $(p s=.05, .11, .11$, and .12) suggested more severe trauma in the debriefing group (i.e., greater percentage of body burned, stressfulness, perceived life threat, and number of days hospitalized, respectively). The authors failed to correct for multiple comparisons, thereby increasing the likelihood of their finding a spurious significant result.

At the initial assessment, although the to-be-debriefed group tended to score slightly higher than the control group on questionnaires measuring anxiety, depression, and posttraumatic stress, the differences were not significant $(p \mathrm{~s}=.77, .43$, and .79, respectively; Bisson et al., 1997). At the 3-month assessment, the rate of PTSD, based on clinical interviews, was nonsignificantly higher in the debriefed group than in the control group ( $21 \%$ vs. $15 \%$ ). At the 13 -month assessment, however, the rate of PTSD was significantly higher among debriefed subjects than among control subjects (26\% vs. 9\%). Moreover, at 13 months, the debriefed group had significantly higher scores on self-report measures of PTSD, anxiety, and depression than did the control group. These significant differ- ences remained even when the researchers controlled statistically for baseline severity of PTSD, anxiety, and depression. Worse outcomes were associated with longer debriefing sessions, more severe burns, higher initial scores on a psychopathology questionnaire, and a shorter period of time between the burn trauma and the debriefing session. Nevertheless, $52 \%$ of the participants endorsed debriefing as "definitely useful" (Bisson et al., 1997, p. 79). The authors, however, concluded that even if debriefing is merely ineffective, rather than harmful, "its routine use should be discontinued" (p. 81). That is, continued use of an inert (and possibly harmful) intervention is a waste of time and resources, and impedes discovery of early interventions that actually do reduce risk of subsequent psychopathology.

In the second study showing that debriefing may have harmful effects, Hobbs, Mayou, Harrison, and Worlock (1996) randomly assigned victims of road traffic accidents to either a single debriefing session ( $n=54$ ) or an assessment-only control condition $(n=52)$. Victims received individual, one-onone debriefing, not group debriefing. Despite randomization, the debriefing group had a higher score on an index of injury severity. The groups did not differ in terms of baseline severity of PTSD or other psychiatric symptoms. The 1-hr debriefing occurred between 24 and $48 \mathrm{hr}$ after the accident, and involved providing information about common emotional reactions, reviewing the trauma, encouraging emotional expression, and suggesting gradual return to normal travel. At the 4-month assessment, neither group evinced a reduction in symptoms of PTSD, anxiety, or depression. Moreover, the debriefing group had significantly worse scores than the control group on two subscales of the Brief Symptom Inventory (a questionnaire tapping symptoms of emotional disorder). However, 4-month follow-up data were unobtainable for $22 \%$ of the debriefing group and $6 \%$ of the control group.

This research team reassessed the patients from the original study (Hobbs et al., 1996) 3 years later (Mayou, Ehlers, \& Hobbs, 2000). Relative to the control group, the debriefing group reported significantly more PTSD symptoms, general psychiatric symptoms, fear of traveling as a passenger, pain, physical problems, and financial problems. Further analyses indicated that those participants who had initially scored high on the measure of PTSD and were not debriefed improved markedly by the 3-year follow-up. However, those who had originally scored high on the PTSD measure and were debriefed remained highly symptomatic at follow-up. Therefore, debriefing appeared to impede natural recovery from acute PTSD symptoms. Controlling statistically for differences in injury severity, Mayou et al. found that this could not account for the significantly worse outcome of debriefed subjects with high PTSD scores at baseline. This study suggests that individual debriefing may have long-term adverse effects, although the study had limitations, such as a very early intervention, an inability to contact and assess all participants at follow-up, and differences in injury severity between the debriefed and nonde- 
briefed groups. Between-group differences in injury severity complicate interpretation of the results. That is, despite the authors' attempt to statistically control for the effect of differential injury severity, it is difficult to rule out completely that the greater injury severity contributed to the poorer outcome in the debriefed group.

Although the RCT is the gold standard for assessing debriefing or any other intervention, RCTs conducted to date have all concerned one-on-one debriefing, not group debriefing. In the studies we review next, although researchers were unable to assign participants to conditions randomly, chance factors usually determined whether a person received debriefing or not (e.g., whether the person was present at work on the day of the debriefing). These non-RCTs do have the virtue of testing a group debriefing format versus a control (no-treatment) condition.

Hytten and Hasle (1989) assessed 39 volunteer firefighters who had undergone a formal debriefing after having fought a major blaze in a hotel in Norway. All but 1 firefighter considered the intervention helpful. There was no significant difference in self-reported PTSD symptoms between the debriefed group and nondebriefed volunteers who opted to discuss their experience informally with their peers. However, the level of PTSD symptoms was low overall.

Following the Newcastle earthquake in Australia, Kenardy et al. (1996) assessed disaster workers and volunteer helpers who had either been debriefed $(n=62)$ or not $(n=133)$. The groups did not differ in either self-reported exposure to threat or postintervention PTSD symptoms. Although $80 \%$ of the debriefed group found the process helpful, responses on a general health questionnaire indicated that this group was significantly more symptomatic than the nondebriefed group. Unfortunately, there was no random assignment to groups and no verification that the debriefing protocol was implemented properly.

Using Mitchell's (1983) protocol, Carlier, Lamberts, van Uchelen, and Gersons (1998) provided a single session of group debriefing to 46 police officers who had responded to a plane crash. Each group session had a maximum of 10 participants. The debriefers had received formal training in the Mitchell method, and fidelity to protocol was checked. No predebriefing measures were taken, but structured interviews were done 8 and 18 months after the debriefing. A group of 59 police officers who, by chance, had been unable to attend a debriefing session because of schedule conflicts were designated as the comparison group and assessed as well. Although assignment to groups was not random, the groups did not differ in age, sex, history of previous traumas, activities at the disaster site (e.g., body handling, rescue operations), or desire for debriefing. Although $7 \%$ of the total sample had developed acute PTSD shortly after the disaster, by the 8-month assessment, only 2 participants had PTSD; 1 had been debriefed and the other had not. Assessment of PTSD symptoms showed no significant differences between the groups at 8 months. At 18 months, debriefed participants had significantly more PTSD hyperarousal symptoms than did nondebriefed participants, but otherwise the groups were indistinguishable in terms of symptoms.

\section{Debriefing Advocates Respond to the Negative Studies}

Studies adduced in support of debriefing are marred by serious methodological flaws. And almost all well-designed studies have failed to confirm the efficacy of debriefing as a means of preventing posttraumatic psychopathology. However, advocates of debriefing argue that these negative studies are characterized by fatal flaws that undermine their probative import. We next scrutinize the merits of these critiques.

\section{Group versus individual debriefing}

RCTs have failed to demonstrate that debriefing reduces subsequent psychopathology, and two trials have shown that debriefing may impede natural recovery from trauma. However, none of these RCTs employed group debriefing. According to Everly and Mitchell (1999), such studies do "not warrant consideration" (p. 125).

There are several problems with Everly and Mitchell's dismissal of RCTs on individual-format debriefing. First, "in clinical practice individual debriefing is the rule rather than the exception" (van Emmerik et al., 2002, p. 767). Second, debriefing advocates (e.g., Mitchell, 2002) approvingly cite oneon-one debriefings if they regard the results as favorable (e.g., Campfield \& Hills, 2001; Flannery et al., 1991). It is unclear why these studies are deemed probative, whereas individualformat studies yielding disappointing results are not. Third, it is unclear why an intervention would be inert (or harmful) when delivered in a one-on-one format, but efficacious if delivered in the presence of other people. A group may mobilize social support, but it may also inhibit frank expression of thoughts and feelings, especially among one's coworkers. A further risk in group debriefing is the possibility of vicarious traumatization. Listening to the gruesome details of the event experienced by coworkers may worsen group members' own distress. Fourth, if group debriefing were, in fact, efficacious, then nonrandomized studies comparing groups of debriefed subjects with matched groups of nondebriefed subjects should confirm the superiority of group debriefing. However, such studies have indicated either no effect on PTSD symptoms (e.g., Hytten \& Hasle, 1989) or slightly adverse effects (e.g., Carlier et al., 1998; but see Wee et al., 1999, for a possible exception).

\section{Debriefing as a stand-alone technique}

Everly and Mitchell (1999) have claimed that CISD was never "meant to be a stand alone technique" (p. 93). There are several problems with this criticism, however.

First, according to Mitchell (1983), a single CISD session "will generally alleviate the acute stress responses which ap- 
pear at the scene and immediately afterwards and will eliminate, or at least inhibit, delayed stress reactions" (p. 36). Indeed, after conducting a meta-analysis of several nonrandomized studies (all included in our previous discussion) in which CISD was a single, stand-alone intervention, Everly et al. (1999) affirmed "the power of the psychological debriefing technology to mitigate symptoms of psychological distress" (p. 232). Second, as Rose et al. (1999) observed, debriefing is almost always applied as a single-session intervention rather than as a component in some larger package. Third, Carlier et al. (2000) administered three spaced debriefing sessions, and found small, but adverse, effects. Fourth, debriefing advocates often claim that researchers must test CISM rather than merely CISD - one of its components. However, CISM is not an intervention at all. It is an umbrella term embracing diverse techniques that are relevant to different contexts. CISM includes activities ranging from in-service continuing education programs to outreach support programs for families whose loved ones perished in the line of duty to programs providing coffee and doughnuts to relief workers at disaster sites. Hence, unlike CISD, CISM is not a crisis intervention itself, but rather an administrative framework.

\section{Debriefed people appreciate debriefing}

Debriefing advocates correctly state that most debriefed people appreciate the experience. This is true even in studies showing adverse effects of debriefing (e.g., Bisson et al., 1997; Carlier et al., 2000). Therefore, we believe that consumers' satisfaction ratings apparently reflect polite expressions of gratitude rather than intervention efficacy.

Furthermore, there are other possible explanations for the perceived helpfulness of debriefing. For example, people usually feel better at follow-up and may attribute this to the debriefing, not knowing that, on average, they likely would have been just as well if they had not been debriefed. If they had this information, their helpfulness ratings may well be different. Also, justification of effort is a well-known psychological phenomenon. When people are made to comply with unpleasant tasks, they later rate those tasks more positively the less external justification they had for doing them.

\section{Researchers have used the wrong measures to evaluate debriefing}

According to Mitchell (2002), researchers who have reported negative outcomes have made the "egregious" (p. 18) error of "using treatment outcome measures (dependent variables such as reductions in clinical depression and symptoms of Posttraumatic Stress Disorder) instead of crisis intervention outcome measures (adaptive function, return to work, lower sick time utilization). That very fact indicates that the researchers have confused crisis intervention and psychotherapy" (p. 18).

There are several problems with this objection. First, using measures of PTSD to evaluate the preventive impact of a crisis intervention does not mean that researchers have confused crisis intervention with psychotherapy. (Ironically, in view of this objection, Mitchell, 2002, himself cited as supportive of debriefing studies of treatments that fail to count as crisis interventions. For example, the intervention studied by Chemtob et al., 1997, was delivered long after the trauma, and the intervention studied by Busuttil et al., 1995, was integrated into a residential group psychotherapy program). Second, elsewhere he stated that the goal of his approach is "to reduce the incidence, duration, and severity of, or impairment from, traumatic stress" (Everly \& Mitchell, 1999, p. 72). Accordingly, researchers have assessed PTSD symptoms as an index of impairment from traumatic stress. If crisis intervention following traumatic stressors is a form of secondary prevention, then it ought to prevent the emergence of PTSD. Third, Mitchell (2002) does not object to using PTSD symptoms as an outcome measure when he believes that the study shows favorable effects of debriefing (e.g., Chemtob et al., 1997; Wee et al., 1999). It is unclear why measurement of PTSD symptoms is appropriate when the results appear favorable, but inappropriate when the results appear unfavorable.

\section{Inappropriate participants}

Mitchell (2002) criticized certain debriefing studies for including primary victims of trauma (i.e., not emergency service personnel), but he directed this critique at studies with negative outcomes only and cited studies on primary victims approvingly when he believed the results supported his approach (e.g., Amir et al., 1998; Campfield \& Hills, 2001; Wee et al., 1999). This seems inconsistent: If debriefing advocates believe that primary victims should be excluded from studies on the efficacy of debriefing, then they should not support the efficacy of debriefing by citing studies on primary victims that yielded results they deem favorable.

\section{Other departures from protocol}

When researchers fail to confirm the efficacy of debriefing in controlled studies, debriefing advocates reply that proper protocol was not followed. Negative studies can then be dismissed as irrelevant.

There are several problems with this criticism. First, it presupposes the efficacy of the specific protocol. To say that a departure from recommended protocol, such as failing to debrief in groups, is responsible for the null results, one must first document that the specific protocol is, indeed, effective. Debriefing advocates seemingly believe that one is entitled to assert the efficacy of debriefing until scientists "prove" that it does not work. This logic is exactly backwards: The burden of proof lies squarely on the shoulders of those claiming the efficacy of a specific protocol. Only when a specific protocol has been shown to be effective is one entitled to complain when researchers depart from it. 
Second, in most of the negative studies, the researchers did use the "Mitchell model" of debriefing, albeit often with oneon-one debriefings. And some of the studies cited in support of CISD depart even more dramatically from the recommended protocol (Busuttil et al., 1995; Chemtob et al., 1997) than do the RCTs on individual debriefing (Bisson et al., 1997).

\section{CONSIDERATIONS FOR CRISIS INTERVENTION}

\section{The Right Time to Talk About the Trauma}

Studies showing null effects for psychological debriefing motivate reexamination of a belief shared by many trauma specialists: that expressing thoughts and feelings about the trauma hasten healing, and that "bottling up" these feelings will impede recovery. Some evidence supports this view. Pennebaker and his colleagues have found that repeated writing about one's thoughts and feelings concerning a very upsetting personal event has positive long-term effects on one's mood and health (e.g., Pennebaker \& Beall, 1986). Conversely, attempts to avoid thinking about one's trauma and to avoid reminders of trauma are associated with persistent PTSD symptoms (e.g., Ehlers et al., 1998). Furthermore, most trauma therapies emphasize the importance of talking about one's feelings and thoughts about the trauma.

These research findings seem to suggest that helping people ventilate their emotions soon after a critical event will hasten recovery from posttraumatic stress. However, the problem with this inference is that this research was done weeks, months, or years after the trauma, and thus may not apply to the immediate aftermath of an event. Indeed, as Pennebaker (2001) emphasized, his research focused on the psychobiological benefits of writing about traumatic events that had remained undisclosed for months or years. Hence, Pennebaker's work cannot be adduced in support of psychological debriefing that occurs shortly after the traumatic event.

What do people (most of whom will recover on their own) actually do to process a traumatic event? They appear to alternate between phases of avoidance and phases of processing (e.g., Horowitz, 1986; Pennebaker \& Harber, 1993). Furthermore, if given a choice, only about $10 \%$ of trauma survivors seek to discuss their experience with mental health professionals (e.g., Rose et al., 1999). In the days and even weeks after a traumatic event, "an individual may or may not be in a state in which he or she wishes, or is prepared, to discuss what has happened" (Raphael, Wilson, Meldrum, \& McFarlane, 1996, p. 466).

Professionals working with trauma survivors may have too quickly concluded that the initial disinclination of survivors to discuss their trauma constitutes a form of dysfunctional avoidance likely to hinder recovery. The intermittent processing favored by most survivors may adaptively enable them to begin rebuilding their lives and to concentrate on the practical prob- lems they face, and thereby help them to put the event in the past. Furthermore, memories tend to fade with time, and it remains untested whether very early exposure to traumatic memories promotes or retards this process. Research has shown that certain conditions are necessary to facilitate emotional processing of distressing material: "The material, especially in the early stages of treatment, should be made predictable, controllable, presented in small chunks, and tackled in a progressive but gradual way" (Rachman, 2001, p. 166). These conditions are seldom met in the immediate aftermath of trauma. Thus, encouraging survivors to discuss their thoughts and feelings right away may increase the risk that they will be overwhelmed by the experience, which will be counterproductive. Furthermore, as Rachman (2001) has pointed out, there are several routes to emotional processing, and the activation of the trauma memory by reliving the experience may be only one of them.

Thus, contrary to a widely held belief, pushing people to talk about their feelings and thoughts very soon after a trauma may not be beneficial. Perhaps systematic exposure to the trauma memories should be reserved for people who fail to recover on their own. Similarly, Brewin (2001) concluded that

any intervention that is carried out within two or three days following a mild trauma, or within a month following a severe trauma, is probably coinciding with natural recovery processes. An obvious concern is that the intervention should interfere as little as possible with these processes, at least until some hindrance of recovery is evident. (p. 166)

Thus, clinicians working with trauma survivors soon after the event face a dilemma. On the one hand, any intervention they offer should not interfere with natural recovery. On the other hand, they will want to offer treatment as soon as possible to those survivors who are unlikely to recover on their own, to shorten their suffering and to prevent the development of secondary problems such as job loss, problems with relationships, or substance abuse. In Identification of Individuals at Risk for Chronic PTSD, we address how best to identify trauma survivors who are unlikely to recover on their own.

The practice of talking about a traumatic event shortly after its occurrence has a long historical tradition in military settings. The principles of proximity, immediacy, and expectancy (PIE) have often governed early intervention in the military (Artiss, 1963). Distressed soldiers are treated close to the battlefield (proximity), as soon as possible (immediacy), and with full expectation that they will return to duty (expectancy). The treatment seldom involves more than providing food, rest, and reassurance that they will be feeling better soon. Although these principles have often been accepted as useful in military contexts, the PIE approach has seldom been evaluated rigorously. In one ambitious study, Solomon and Benbenishty (1986) studied troops involved in the Lebanon War. Some were managed according to PIE principles, and others were treated some distance from the battlefront. Solomon and Benbenishty reported that troops managed according to the principles of PIE 
displayed reduced rates of PTSD 1 year later. The findings cannot be considered definitively supportive of PIE because whereas proximity was objectively defined by the location of the intervention, immediacy and expectancy were operationally defined by requesting soldiers to rate how immediate the intervention was and how much they were expected to return to their unit; unfortunately, these ratings were made 1 year after the treatment was administered. Considering the evidence that memory for initial posttraumatic experiences and responses is strongly influenced by current severity of PTSD symptoms (Harvey \& Bryant, 2000a; Schwarz, Kowalski, \& McNally, 1993; Southwick, Morgan, Nicolaou, \& Charney, 1997), it is likely that soldiers' retrospective ratings were influenced by their current symptoms.

\section{Survivors' Needs in the Aftermath of Trauma}

We are not arguing that mental health professionals should leave trauma survivors alone in the immediate aftermath of trauma. Indeed, perceived lack of social support is strongly linked to heightened risk for PTSD (Brewin et al., 2000). Thus, assessing and, if necessary, facilitating social support may promote recovery from trauma. Many survivors have good support networks and may prefer to rely on their trusted confidants, but others may need help in activating social support because they do not have access to good support (whether because of the loss or separation from significant others, preexisting poor support, or the perception that previously trusted people do not understand their plight). Recent recommendations for crisis intervention programs (e.g., Litz et al., 2002; Raphael \& Dobson, 2001) take into account that the posttrauma environment has an important influence on recovery and urge that social support be facilitated (including by trying to increase community cohesion if an entire community is affected; Meichenbaum, 1994). Controlled evaluations of such efforts are lacking, however. Dunmore, Clark, and Ehlers (1999, 2001) found that the perception of negative social interactions with others in the aftermath of trauma predicted chronic PTSD to a greater extent than did lack of perceived positive support. This implies that sensitive, respectful attitudes on the part of emergency, hospital, and police staff may help buffer survivors against developing PTSD.

As Raphael et al. (1996) observed, "The provision of practical help may ultimately be seen as more helpful and positive than the specific psychological care offered" (p. 466). Trauma survivors have many different immediate needs in their efforts to adjust to the event. Their needs will depend on the kind of trauma they have experienced (e.g., individual traumas vs. disasters), the nature and extent of their physical injuries, the nature and extent of other losses they are facing (e.g., loss of housing and loved ones), and their emotional responses to the event. For example, survivors may need immediate comfort, reassurance, and help establishing safety (e.g., housing); help to overcome extreme fatigue and exhaustion; financial support; help finding relatives and friends; time to themselves to come to terms with what happened, and therefore practical help with child care or other duties; and advice and support to cope with the additional burdens caused by the aftermath of the trauma (e.g., Raphael et al., 1996; Ursano, Grieger, \& McCarroll, 1996).

Many survivors will need information, such as information about what exactly happened during the event and to their relatives, or what their chances of recovery from serious injuries are (see also Brewin, 2001). Although provision of information alone does not appear to promote recovery (Brewin, 2001; Rose et al., 1999), it is generally recommended that survivors be provided information about common reactions to trauma, including natural recovery. This information should acknowledge the magnitude of the trauma and reassure survivors that it is normal to have symptoms of PTSD in the aftermath of a traumatic event.

Crisis intervention methods that sensitively focus on the individual's needs are currently under discussion, and empirical data are largely lacking (see Orner \& Schnyder, in press). Current guidelines usually recommend a range of immediate measures under the umbrella term of "psychological first aid" (e.g., Litz et al., 2002; Raphael et al., 1996). This includes

the basic human responses of comfort and consoling a distressed person; protecting a person from further threat or distress, as far as is possible; furnishing immediate care for physical necessities, including shelter; providing goal orientation and support for specific realitybased tasks ("reinforcing the concrete world"); facilitating reunion with loved ones from whom the individual has been separated; facilitating some telling of the "trauma story" and ventilation of feelings as appropriate for the particular individual; linking the person to systems of support and sources of help that will be ongoing; facilitating the beginning of some sense of mastery; and identifying the need for further counseling or intervention. (Raphael et al., 1996, pp. 466-467)

Some of these goals overlap with those of CISM. As in psychological debriefing, the ventilation of feelings and telling of the trauma story is recommended with the proviso that it should be appropriate for the particular individual. Thus, psychological first-aid respects an individual's wishes regarding whether to talk about the trauma.

Litz et al. (2002) described how psychological first aid deals with the issue of whether or not trauma survivors should be encouraged to talk about the traumatic event in its immediate aftermath:

Individuals who choose not to participate in groups should be given the opportunity to meet with individual therapists with trauma expertise and experience. Those survivors not interested in any formal intervention should be asked if they care to discuss their thoughts and feelings about the event and urged (if possible) to voice their ideas about the personal implications of the experience to significant others when they feel most comfortable doing so. The goal is not to maxi- 
mize emotional processing of horrific events, as in exposure therapy, ${ }^{5}$ but rather to respond to the acute need that arises in many to share their experience, while at the same time respecting those who do not wish to discuss what happened. (p. 128)

Foa (2001) suggested that in the immediate aftermath of trauma, people should follow their natural inclination with regard to how much and to whom they talk, and that professionals should listen actively and supportively, but not probe for details and emotional responses or push for more information than survivors are comfortable providing.

The bottom line is that in the immediate aftermath of trauma, professionals should take their lead from the survivors and provide the help they want, rather than tell survivors how they will get better. As Raphael and Dobson (2001) pointed out, "There has been a failure in many formats of acute posttrauma intervention to develop and utilize a systematic, scientifically based, and clinically appropriate framework of assessing need" (p. 153). Given present knowledge, it is impossible in the immediate aftermath to tell which survivors will later need psychological treatment.

It remains to be tested empirically whether psychological first aid is effective in promoting recovery from posttraumatic stress. As the debate about psychological debriefing has shown, plausible ideas about what interventions make sense in the aftermath of trauma do not necessarily mean that these interventions will promote recovery from posttraumatic stress. Raphael and Dobson (2001) arrived at a similar conclusion, noting that although psychological first-aid interventions "are intended to be generic and supportive, they have not been subjected to research and evaluation, so that the usefulness and validity of their application needs to be established. Their general supportive nature and nonactive intervention suggest that they are unlikely to do harm" (p. 143).

It is interesting that the consensus opinion appears to be returning to views that prevailed in military circles 50 years ago. During World War II, American officers held group debriefing following combat, and the process was conceptualized as a review and reconstruction of the event in which the perspectives of all participants were validated nonjudgmentally (for a review, see Shalev, 2000). Advice, interpretation, or other direc-

5. Exposure therapy for PTSD is a behavioral treatment that helps the person confront trauma memories and reminders of the event that evoke intense emotional or physical responses. It involves emotional and detailed recounting of the traumatic memories in the temporal order in which the event (or events) unfolded. The recounting includes the person's thoughts and feelings. Recounting is done either by visualizing the event in one's imagination and talking about what one visualizes (imaginal exposure) or by writing a detailed account of the traumatic event. The recounting is usually repeated until it no longer evokes high levels of distress. In addition to imaginal reliving, exposure often entails an in vivo (real-life) component in which patients enter situations or engage in activities associated with the trauma until the stress diminishes. For example, a survivor of a motor vehicle accident may practice driving his or her car past the scene of the accident until distress associated with the memories of the accident subsides. tive interventions were not provided. History has turned full circle in that trauma counselors are again recognizing that approaches that are supportive and noninterventionist may be optimal in the immediate aftermath of trauma. It appears that the focus is shifting from directly encouraging people to review and disclose their experiences (reflected in CISD) to providing support and a forum for people to discuss their reactions if they are so inclined.

\section{IDENTIFICATION OF INDIVIDUALS AT RISK FOR CHRONIC PTSD}

As we discussed earlier, the majority of people exposed to trauma will experience transient stress reactions that remit within 3 months of the traumatic event. If mental health resources are allocated to those who will experience a chronic mental disorder, an important goal for mental health professionals in the acute posttrauma phase is to identify individuals who will develop a chronic disorder. That is, there is a need to identify people who will subsequently develop a chronic disorder because this subset of trauma survivors, unlike those who experience a transient stress reaction, will require treatment. This identification procedure has been termed the "triage" (Raphael et al., 1996) or "screen and treat" approach (Brewin, 2001).

There are important reasons for screening trauma survivors before providing an intervention. First, one has to bear in mind that traumatic events can trigger not only PTSD, but also a range of other disorders, such as psychosis. Second, the purpose of screening is to identify those survivors who are unlikely to recover on their own and therefore in need of treatment. Prospective longitudinal research has identified predictors that can be used for this task. Current research indicates that the single most important indicator for the risk of chronic PTSD is the severity of PTSD symptoms. Although symptom severity in the initial days after a trauma is not a good indicator of PTSD risk (Shalev, 1992), from about 1 to 2 weeks after the event onward, the number of symptoms, their severity, or both predict chronic PTSD (Harvey \& Bryant, 1998b; Koren et al., 1999; Murray et al., 2002; Shalev et al., 1997). Brewin (2001) recommended carefully monitoring symptoms in the aftermath of the event, preferably with validated screening instruments. He recommended intervention only when symptoms fail to subside naturally by about 4 to 6 weeks posttrauma. Schnyder and Moergeli (in press) emphasized that a single screening may be insufficient because a certain number of people will have a delayed onset of chronic PTSD symptoms.

Practitioners need economical instruments for screening large populations of survivors to identify those at risk for chronic PTSD. Brewin et al. (2002) have developed a promising screening questionnaire. It identifies PTSD by any combination of six reexperiencing or hyperarousal symptoms and has excellent agreement with clinician diagnoses of PTSD. Although this instrument shows promise for screening for chronic 
PTSD, there is a need for validated screening instruments that can be used with individuals recently exposed to trauma, so that those who will subsequently develop chronic PTSD can be identified; prospective longitudinal studies are warranted to develop these instruments.

Another indicator relevant to early identification of people who will develop chronic PTSD is depression. In one study, survivors who had major depression in addition to PTSD at 1 month after the event showed greater decreases in their ability to function at work and with friends and family and had a greater chance of having PTSD at 4 months than did those who had PTSD without depression (e.g., Shalev, Freedman, et al., 1998).

The ways people try to cope with trauma are also relevant. One possible indicator of need for early treatment is rumination (e.g., going over and over in one's mind questions like "Why did the trauma happen to me?" "How could I have prevented this from happening?" "What if I had done X?" and "What would my life be like if this had never happened?"). Taking rumination into consideration, in addition to severity of initial symptoms, improves predictions of who will get chronic PTSD (Ehlers et al., 1998; Murray et al., 2002). Similarly, excessive precautions, such as sleeping only with a knife near one's bed and with the lights on (Dunmore et al., 2001), and excessive avoidance, such as not leaving one's house (Bryant \& Harvey, 1998), are associated with risk for persistent PTSD.

The way trauma survivors interpret the initial posttrauma symptoms, such as reexperiencing, numbness, and irritability, predicts the persistence of symptoms independently of symptom severity (Dunmore et al., 2001; Ehlers et al., 1998). Survivors who interpret these symptoms as signs that they might be going crazy, about to lose control, or permanently changed for the worse are at greater risk for chronic symptoms and in greater need of treatment than are those who interpret their symptoms as a normal part of recovery. Sadly, many trauma survivors endure long-lasting physical consequences, such as chronic pain, visible scars, or loss of limbs. These survivors have a greater chance of having chronic PTSD and thus a greater need for help than those who are unhurt or who recover well from their physical injuries (Blanchard et al., 1997; Ehlers et al., 1998).

Thus, although research has not confirmed optimal criteria for establishing an individual's need for early treatment, the results from prospective longitudinal studies have suggested indicators that may aid detection of individuals unlikely to recover without treatment. Future research may show that a particular combination of measures is better in predicting low chance of recovery than is symptom severity alone. For example, Halligan, Michael, Ehlers, and Clark (2003) conducted a prospective longitudinal study of assault survivors and found that a combination of assault severity and cognitive measures predicted $71 \%$ of the variance of PTSD symptom severity at 6 months after the trauma, whereas initial symptom severity predicted $55 \%$ of the variance. From a practical point of view, the severity of the early posttrauma symptoms from about 1 to 2 weeks after the trauma onward is currently the most straightforward indicator of need for treatment. (Note that this assumes that the trauma survivor is safe when the symptoms are assessed).

\section{EARLY TREATMENT OF SURVIVORS WITH CLINICAL SYMPTOMS OF POSTTRAUMATIC STRESS}

\section{CBT Starting in the First Month After Trauma}

Unlike debriefing, psychological treatments for PTSD symptoms in the initial weeks and months after trauma were mainly adapted from CBT programs for PTSD (e.g., Foa \& Meadows, 1997; Foa \& Rothbaum, 1998). Much as in the case of psychological debriefing, early attempts to apply CBT techniques in the first few weeks following rape failed to demonstrate efficacy (Frank et al., 1988; Veronen \& Kilpatrick, 1983). Although the patients receiving CBT showed substantial improvement in psychological symptoms, the studies did not establish that these changes were greater than those occurring with natural recovery. Furthermore, these studies had methodological problems that made their interpretation difficult. However, more recent studies, including several RCTs, suggest that CBT may be effective in treating PTSD symptoms and thus speeding up recovery in people recently exposed to trauma, and some of the studies have shown that early CBT treatments reduce the risk of long-term PTSD (see reviews by Ehlers \& Clark, 2003; Litz et al., 2002). In contrast to the studies of debriefing, the CBT studies have focused on individual treatment.

Using a CBT approach, Foa, Hearst-Ikeda, and Perry (1995) treated 10 female victims of rape or aggravated assault, most within several weeks after the trauma. All met symptomatic, but not duration, criteria for PTSD. The intervention comprised four weekly 2-hr sessions that included treatment elements that have figured prominently in subsequent RCTs: education about trauma symptoms, detailed reliving of the traumatic event in memory, real-life exposure to avoided situations associated with the assault, cognitive restructuring designed to modify maladaptive beliefs, and training in relaxation and breathing skills. Ten other assault victims received only repeated assessments. Unfortunately, victims were not randomly assigned to the treatment versus assessment-only conditions.

The treatment Foa et al. (1995) provided proceeded as follows. During the first session, the therapist educated the patient about typical acute responses to trauma, and assembled a list of objectively safe situations and activities that the patient had been avoiding since the assault. During the second session, the therapist furnished a rationale for exposure therapy, emphasizing that many symptoms continue to occur because the patient has not adequately processed the trauma. After teaching the patient deep muscle relaxation and controlled breathing skills, the 


\begin{abstract}
therapist asked the patient to close her eyes and describe the assault in the present tense as if it were happening again (imaginal exposure). As the patient repeatedly relived the traumatic event, the therapist took note if she expressed any cognitive distortions regarding the excessive dangerousness of the world or her own perceived incompetence. Both the relaxation procedure and the imaginal reliving were audiotaped, and the patient was instructed to listen to the tapes as homework practice. The patient was also encouraged to confront avoided situations and activities (exposure in vivo). Finally, the therapist initiated a discussion of the irrational beliefs expressed by the patient during the imaginal reliving. The third session began with $45 \mathrm{~min}$ of imaginal exposure followed by further cognitive therapy designed to correct distorted beliefs. Chief targets included the patient's beliefs about the unpredictability, uncontrollability, and dangerousness of the world, as well as any extremely negative beliefs she expressed about herself. The therapist helped the patient identify these problematic beliefs, and the patient's homework was to address negative thinking in everyday life. The fourth session included imaginal exposure, cognitive restructuring, and a review of skills mastered by the patient in the program.
\end{abstract}

In addition to the pretreatment assessment, Foa et al. (1995) administered assessments at 2 months and 5.5 months postassault. At 2 months, fewer treated than untreated patients met criteria for PTSD (10\% vs. $70 \%)$. Relative to untreated patients, treated patients reported significantly fewer reexperiencing and arousal (but not avoidance) symptoms of PTSD. However, both groups continued to improve, and at the 5.5month assessment, the untreated and treated patients did not differ significantly on measures of PTSD. This pilot study suggests that CBT may accelerate natural recovery from trauma.

Encouraged by these findings, Foa, Zoellner, and Feeny (2002) conducted an RCT involving survivors of physical or sexual assault who met symptomatic criteria for PTSD in the initial weeks after the event (range: $2-46$ days, mean: 21 days). Patients were randomly assigned to receive four weekly 2-hr sessions of CBT $(n=31)$, repeated assessment $(n=30)$, or supportive counseling $(n=29)$. The third group was added 1 year into the study.

Unlike in the previous study, patients in the repeated-assessment condition improved just as much as did those in the CBT condition (Foa et al., 2002). Among those patients who completed the study, at posttreatment (i.e., when treatment ended) the proportion of CBT-treated patients who had PTSD was $45 \%$, whereas the proportion of repeated-assessment patients who had the disorder was 55\%-a nonsignificant difference. The supportive-counseling group had similar PTSD rates at posttreatment (52\%), but reported greater PTSD severity and greater general anxiety than the CBT group. Thus, there was some evidence that CBT led to greater improvement in the severity of symptoms than did supportive counseling.

Follow-up assessments, conducted about 9.5 months later, did not alter the picture (Foa et al., 2002). There were no differ- ences among the groups in the proportion of patients who still had PTSD (CBT, 32\%; repeated assessment, 30\%; supportive counseling, 29\%). The positive outcome of the assessment group is noteworthy, although it is not clear why this study and the previous one (Foa et al., 1995) had different patterns of results for the assessment-only groups. The assessment conditions in the studies differed somewhat in that patients in the Foa et al. (2002) study received longer clinical assessments (that lasted as long as the sessions of the CBT and supportivecounseling conditions) than patients in the assessment condition in the Foa et al. (1995) study. The pattern of results from these studies raises several possibilities: (a) It is conceivable that detailed assessments with an empathic clinician have a positive impact on recovery. (b) For unknown reasons, the sample of the Foa et al. (2002) study may have been less responsive to CBT than the sample of the early study. (c) Early supportive counseling may impede rather than promote recovery from trauma.

\section{CBT for ASD}

While Foa's group in Philadelphia was testing methods for treating posttrauma symptoms soon after the event, Bryant and his colleagues were doing likewise in Sydney, Australia. The chief difference between the two research programs is that Foa targeted trauma survivors meeting symptomatic criteria for PTSD, whereas Bryant targeted those who met criteria for ASD. Thus, in the Australian studies, only patients who showed significant dissociative symptoms in addition to their PTSD symptoms were included.

In their first study, Bryant's group randomly assigned survivors of industrial or motor vehicle accidents to either CBT $(n=$ 12) or supportive counseling ( $n=12$; Bryant, Harvey, Dang, Sackville, \& Basten, 1998). All patients met criteria for ASD, and treatment commenced within 2 weeks of the accident. Both interventions consisted of five weekly 1.5 -hr sessions of individual therapy. CBT included education about common posttraumatic reactions, progressive muscle relaxation training, imaginal exposure to the traumatic memory, cognitive restructuring of distorted trauma-related beliefs, and graduated in vivo exposure to avoided situations. Supportive counseling included education about posttraumatic reactions plus training in general problem-solving skills within the context of an unconditionally supportive therapeutic relationship. Posttreatment assessment occurred approximately 42 days after the pretreatment assessment, and the follow-up assessment occurred approximately 6 months posttrauma. At posttreatment, significantly fewer CBT-treated patients (8\%) than counseled patients $(83 \%)$ met criteria for PTSD. This difference remained at follow-up (17\% vs. $67 \%)$. At both time points, patients in the CBT group also reported significantly fewer intrusive, avoidance, and depressive symptoms relative to patients in the counseling group. 
In their second study, Bryant, Sackville, Dang, Moulds, and Guthrie (1999) randomly assigned survivors of motor vehicle accidents or nonsexual assault to five weekly 1.5-hr sessions of (a) combined exposure therapy, cognitive therapy, and anxiety management ( $n=15$ ); (b) combined exposure therapy, cognitive therapy, and supportive counseling $(n=14)$; or (c) supportive counseling $(n=16)$. Exposure therapy in this study combined imaginal and in vivo exposure. All patients met (or nearly met) criteria for ASD, and treatment began within 2 weeks of the trauma. At the end of treatment, fewer patients who had received CBT (exposure, cognitive therapy, and anxiety-management group, 20\%; exposure, cognitive therapy, and supportivecounseling group, 14\%) than who had received supportive counseling only (56\%) met criteria for PTSD. Likewise, at 6 months posttrauma, the proportions meeting criteria for PTSD were $23 \%, 15 \%$, and $67 \%$ in the three groups, respectively. The two groups receiving exposure therapy plus cognitive therapy were indistinguishable on various outcome measures.

These results indicate that a combination of prolonged imaginal and in vivo exposure with cognitive therapy is more effective in preventing PTSD than is supportive counseling. Anxiety management does not enhance these preventive effects. Because as many as $80 \%$ of persons with ASD later develop PTSD (Harvey \& Bryant, 1998b; Murray et al., 2002), the results suggest that supportive counseling may have some preventive power. However, some prospective studies have indicated that the percentage of ASD patients who later develop PTSD may be as low as 30\% (O'Donnell, Creamer, Pattison, \& Atkin, 2001), so it is also possible that supportive counseling impeded natural recovery. The lack of an assessment-only control condition makes it impossible to conclude what effect supportive counseling had, if any. Another limitation of this study is that several patients (not included among those whose results we have reported) dropped out of each of the treatments. The dropouts had significantly more severe ASD than those who completed the trial. A 4-year follow-up study of ASD patients who had received either CBT or supportive counseling (Bryant et al., 1998, 1999) revealed that CBT patients maintained most of their gains and that they were still doing better than patients who had received supportive counseling (Bryant, Moulds, \& Nixon, 2003).

Additional studies from Bryant's group demonstrated encouraging results for five to six sessions of CBT with ASD patients. In one study, civilian trauma survivors $(n=89)$ were randomly assigned to receive $\mathrm{CBT}$, CBT plus hypnosis, or supportive counseling (Bryant, Moulds, Guthrie, \& Nixon, in press). Hypnosis was added to CBT in one group because some scholars believe that hypnosis may be especially helpful for trauma survivors with dissociative symptoms (Spiegel, 1996) and because ASD patients are excellent hypnotic subjects (Bryant et al., 2001). A hypnotic induction and suggestion to engage in the imaginal exposure exercise was administered immediately prior to imaginal exposure therapy in an effort to foster emotional processing. Among those completing the
RCT, fewer patients in either the CBT (13\%) or CBT-plus-hypnosis $(9 \%)$ condition than in the counseling condition $(56 \%)$ met criteria for PTSD at posttreatment. At 6-month follow-up, the numbers were $21 \%, 22 \%$, and $59 \%$, respectively. At posttreatment, patients in the CBT-plus-hypnosis group reported significantly fewer reexperiencing symptoms than did those in the CBT-alone group. There may be value in adding hypnosis to imaginal exposure.

Finally, 24 ASD patients who had sustained a mild traumatic brain injury during a motor vehicle accident were randomly assigned to receive five sessions of either CBT or supportive counseling (Bryant, Moulds, Guthrie, \& Nixon, 2003). ASD patients who sustained a mild brain injury are at risk for PTSD despite having had impaired consciousness during the accident (Bryant \& Harvey, 1998). The proportions of patients meeting criteria for PTSD at the 6-month follow-up were $8 \%$ in the CBT group and $58 \%$ in the counseling group. Thus, loss of consciousness during part of the traumatic event does not impede an ASD patient's ability to benefit from CBT.

In a study yielding data comparable to those of Bryant et al. (2003), Gidron et al. (2001) developed a two-session CBT intervention that was intended to promote memory reconstruction among individuals who had been involved in traffic accidents. The intervention was delivered over the telephone from 1 to 3 days after the accident. Individuals qualified for this RCT if they had a heart rate higher than 94 beats per minute at admission to the emergency room, and were therefore considered at risk for PTSD. Patients who received this intervention $(n=8)$ had greater reductions in severity of PTSD symptoms 3 to 4 months after the trauma than did those who received two sessions of supportive listening over the telephone $(n=9)$.

It is unclear why Bryant's group found much more pronounced differences between early CBT and supportive counseling than did Foa's group. Both research teams treated patients within weeks of the traumatic event, and the two groups used similar techniques. A major difference appears to be the pattern of results for the supportive-counseling condition. In the Bryant et al. studies, patients who received supportive counseling still had significant psychopathology and high PTSD rates at follow-up, whereas in the Foa et al. (2002) study, the majority of patients who received supportive counseling had recovered. One possible explanation for this discrepancy is that the samples Bryant et al. studied were at greater risk for chronic PTSD because they met criteria for ASD. However, in an additional analysis of their data, Foa et al. divided their patients into those who met ASD criteria and those who did not and did not find differential outcomes for patients with and without ASD. Therefore, it is unclear whether dissociative symptoms confer risk for chronic PTSD over and above the risk conferred by severity of PTSD symptoms (e.g., G.N. Marshall \& Schell, 2002). Other possible explanations of the difference are a somewhat smaller responsiveness to CBT in the sample studied by Foa et al. (2002), a differential time course 
of recovery depending on the type of trauma, or somewhat stricter exposure homework assignments in the Bryant et al. studies.

\section{CBT Starting 1 to 3 Months After Trauma}

Three other research groups have delivered broadly similar CBT interventions between 1 and 3 months posttrauma. Ehlers et al. (in press) recruited traffic accident survivors with PTSD about 2 months after the accident and had them complete a 3 -week self-monitoring phase prior to enrolling in a formal CBT trial. Those patients who still had PTSD at the end of this phase were randomly assigned to either up to 12 weekly sessions of CBT $(n=28)$, a self-help condition (one session with a clinician and a self-help booklet; $n=28$ ), and repeated, but infrequent, assessments of PTSD symptoms $(n=29)$. The CBT program emphasized cognitive therapy rather than prolonged, repeated imaginal exposure to traumatic memories (see Ehlers \& Clark, 2000). CBT was superior to self-help and repeated assessment on all measures at posttreatment and at follow-up. The $11 \%$ PTSD rate at 6 months after CBT (i.e., 1 year posttrauma) was lower than the $54 \%$ rate for patients receiving repeated assessments and lower than the $60 \%$ rate for patients in the self-help condition. On most measures, the self-help condition did not differ from the repeated-assessment condition; the only exceptions were that the self-help group had a lower rate of high end-state functioning (a combined measure of PTSD symptoms, anxiety, depression, and disability) and a greater rate of requests for treatment at follow-up.

In another study, Öst, Paunovic, and Gillow (2002) randomly assigned crime victims with PTSD to up to 16 sessions of CBT or to a wait list. Treatment began between 4 and 12 weeks posttrauma. CBT comprised imaginal and in vivo exposure and cognitive restructuring techniques. At the end of treatment, the CBT group was significantly superior to the wait-list group on measures of PTSD symptoms, anxiety, depression, quality of life, and social adjustment. Among patients completing the trial, only $5 \%$ in the CBT group still had PTSD, in contrast to $65 \%$ in the wait-list group.

In an RCT from Spain, Echeburua, de Corral, Sarasua, and Zubizarreta (1996) provided a five-session cognitive stressmanagement program to rape survivors 1 to 3 months after the event $(n=10)$ and compared the effects of this approach with those of five sessions of progressive muscle relaxation $(n=$ 10). There was no untreated group. The CBT program included information about typical responses to rape, cognitive restructuring of negative thoughts and guilt related to the event, training in coping skills such as relaxation and thought stopping, and instructions to gradually confront reminders of the event. No imaginal reliving was included. Both interventions markedly reduced symptoms of PTSD, anxiety, and depression. The cognitive stress-management group did not differ from the relaxation group at the end of treatment, but showed lower PTSD symptoms at the 1-year follow-up.
Overall, CBT treatments delivered 1 to 3 months after a trauma show promising results for survivors with PTSD. Relative to no treatment, CBT promotes recovery from trauma.

\section{CONCLUSIONS, UNRESOLVED ISSUES, AND FUTURE DIRECTIONS}

\section{Effects of Early Interventions}

Although psychological debriefing is widely used throughout the world to prevent PTSD, there is no convincing evidence that it does so. RCTs of individualized debriefing and comparative, nonrandomized studies of group debriefing have failed to confirm the method's efficacy. Some evidence suggests that it may impede natural recovery. For scientific and ethical reasons, professionals should cease compulsory debriefing of trauma-exposed people. In response to the disappointing results for psychological debriefing, crisis intervention specialists recommend psychological first aid, which includes attending to the survivors' individual needs in a nonprescriptive, flexible way. Data on the efficacy of this approach are needed. Raphael and Dobson (2001) recently put the need for the evaluation of acute posttrauma interventions in perspective:

Because of the needs of survivors ("victims") and the often highly charged environments that follow traumatic events, there has been a reluctance to evaluate the interventions applied and at times suggestions that to even think of doing so is wrong because everything provided with such goodwill for those so badly affected must be of benefit. This is further emphasized by public demand and the perceived helpfulness of much that is provided. It is only now-with a growing body of evidence that much may not be of benefit, may be costly without good reason, and may even for some possibly produce harm - that requirements for evaluation can really gain acceptance. It should be clear that any interventions must be accountable and that their outcomes must be systematically evaluated in the shorter and in the longer term. Thus the requirement should be in place and a culture developed to evaluate all acute posttrauma interventions and their effectiveness or otherwise. (p. 155)

The evidence for the efficacy of early CBT treatment in preventing chronic PTSD among symptomatic trauma survivors is mixed, but encouraging. It remains unclear whether CBT given in the first month after trauma is more effective than repeated assessment without formal treatment (Foa et al., 2002), although CBT given from 1 month onward appears superior to assessment alone or no intervention (Ehlers et al., in press; Öst et al., 2002). Early CBT is superior to supportive counselingat least for survivors with ASD (Bryant et al., 1998, 1999, in press).

\section{Unresolved Issues}

Ehlers and Clark (2003) and Litz et al. (2002) have identified unresolved issues in need of further research. First, for several possible reasons, some CBT studies have had much higher 
dropout rates than others. For example, Bryant et al. (1999) and Foa et al. (2002) had dropout rates of $20 \%$ and $29 \%$, respectively, whereas Ehlers et al. (in press) had a dropout rate of $0 \%$. Compared with Bryant's and Foa's research teams, Ehlers et al. relied much less on prolonged imaginal exposure (reliving) of the trauma and much more on cognitive therapy for correcting maladaptive trauma-related beliefs. Cognitive therapy may be less stressful and therefore more acceptable, thereby diminishing dropout rates.

Second, people at high risk for chronic PTSD may require more than four or five weekly sessions. Recent studies in England and in Sweden suggest a dozen sessions may be warranted for some individuals.

Third, most studies have emerged from only a handful of research centers, and most patients have been survivors of road traffic accidents or assaults. There is a need to ensure that positive results can be obtained by researchers who are not also the developers of the intervention, and there is a need to ensure that preventive methods can reduce risk of PTSD arising from other stressors. Along these lines, Ehlers and Clark's program was evaluated in an uncontrolled trial for patients exposed to a terrorist bombing in Northern Ireland (including civilians affected by the bombing and professionals who had attended to severely injured and dying people; Gillespie, Duffy, Hackmann, \& Clark, 2002). Excellent results were obtained by National Health Service professionals who received training from Clark and Ehlers's research team. This implies that cognitive therapy methods can be mastered and effectively applied by clinicians who are not experts on PTSD.

Fourth, it may be time to revisit and reexamine certain assumptions in the trauma field in light of emerging evidence on early intervention. For example, both debriefing advocates and CBT specialists have emphasized that detailed exposure to the memory of the traumatic events, including one's thoughts and feelings, is the (or an) avenue to recovery. However, studies indicate that repetitive imaginal reliving of the trauma may not be the only way to promote recovery (Ehlers \& Clark, 2000) and may not even be necessary, as there appear to be multiple ways of promoting emotional processing (Rachman, 2001).

Fifth, clinical researchers need to recognize that traumatic events give rise to problems other than, or in addition to, PTSD. Accordingly, researchers need to assess whether early interventions reduce subsequent substance abuse, depression, and interpersonal problems, as well as stress reactions per se.

Sixth, the optimal time for psychological treatment of trauma survivors who show symptoms of posttraumatic stress remains unclear, and probably depends on several factors. The research on early CBT interventions has concerned people who experienced individual traumas rather than large-scale disasters. For those exposed to disaster, the optimal time for psychological treatment may be later, as treatment will be viable only when safety and infrastructures are reestablished, enabling a return to everyday life. Once these conditions are met, cognitive-behavioral treatments may be of benefit, as suggested by preliminary uncontrolled studies of exposure therapy for earthquake survivors (Başoğlu, Livanou, \& Şalcıoğlu, in press; Başoğlu, Livanou, Şalcıŏglu, \& Kalender, in press).

Several considerations apply in determining when to provide an intervention. On the one hand, it is important to intervene as early as possible to shorten suffering and prevent secondary problems such as alcohol abuse and adverse effects on social relationships. On the other hand, in the immediate aftermath of a trauma, many survivors have other needs that take priority, such as needs for surgical procedures for physical injuries, reestablishing safety, and reestablishing other aspects of normal everyday life (e.g., going back to work to prevent job loss). Thus, Brewin (2001) emphasized that trauma-focused treatment of refugees is likely to be unhelpful or ineffective while they still have realistic concerns about "the current whereabouts of their loved ones, their own housing and subsistence needs, and the probability that they will be returned to their country of origin" (p. 166). Mental health professionals providing early intervention must also recognize that many trauma survivors experience marked grief, which requires time to allow normal recovery. One also needs to bear in mind that very early interventions may treat some people who would recover on their own, and that the proportion of people who will recover without intervention depends on how severe their initial symptoms are. Thus, both the costs and the benefits of early treatment have to be considered.

Finally, traumatic events are very common, and not enough trained CBT therapists are available to treat all survivors with PTSD, let alone respond to major disasters that may affect thousands of people at the same time. It is therefore important to explore other ways of delivering these treatments. Self-help booklets have yielded disappointing results (Ehlers et al., in press). A pilot study of an Internet-delivered CBT treatment yielded promising results, although careful screening of who would be suitable for such approach is warranted (Lange, van de Ven, Schrieken, \& Emmelkamp, 2001).

\section{The Social Context of Intervention}

Early intervention for trauma survivors occurs in a social, political, and economic context. As Amir et al. (1998) observed, offering debriefing and other early interventions for trauma survivors may meet "some social and political needs but not necessarily . . . the needs of the victims" (p. 241). Not everyone exposed to trauma either wants or needs professional help. Many therapists are inclined to attribute reluctance to partake of psychological services to "denial" or "avoidance." But trauma survivors who decline professional help may be either resilient or relying on the family and community networks of social support on which people have traditionally relied (see Gist \& Lubin, 1999).

Interventionists must tread lightly in the wake of disaster so as not to disrupt natural social networks of healing and support (Gist \& Devilly, 2002; Herbert et al., 2001), especially when 
aiming to aid victims in the developing world (Bracken \& Petty, 1998). For example, in many war-torn regions, the main goal of the victims is first to establish safety and then to restore their community and culture, not to process traumatic memories about the past (e.g., Giller, 1998). Offering Western interventions - whether psychological debriefing or CBT—is likely to puzzle the intended beneficiaries, who often regard psychotherapy as utterly foreign to their experience of the world.

Social and cultural factors may also impede natural healing. Certain norms and beliefs may lead survivors to think that they are irreversibly damaged by the trauma, thereby increasing their risk for PTSD. For example, many Kosovar women who were raped during the recent Balkan conflict regarded other people's response to their trauma-namely, the belief that they were defiled by the experience-as the worst part of their rape trauma. Culturally based beliefs that worsen the implications of a trauma may complicate treatment.

Finally, many people believe that experiencing, expressing, and disclosing intense emotion in response to stressors is an adaptive, healthy mode of coping. According to this view, "repressing," or inhibiting, emotional experience and expression is potentially damaging. However, these widely accepted assumptions about emotional processing are coming under increasing critical empirical scrutiny. For example, researchers who have studied modes of coping with everything from surviving a heart attack (Ginzburg, Solomon, \& Bleich, 2002) to experiencing the death of loved ones (e.g., Bonanno \& Kaltman, 1999; Stroebe, Stroebe, Schut, Zech, \& van den Bout, 2002; Wortman \& Silver, 1989) have reported data that either fail to support or contradict these assumptions. And some of this work affirms impressive levels of resilience in the face of irrevocable loss (Bonanno et al., 2002).

\section{The Economic and Legal Aspects of Intervention}

It is impossible to understand the intense controversy regarding early intervention without considering the economic aspects of the debate. As Deahl (2000) wrote,

Many workers in the field of psychological trauma clearly have powerful vested interests in promoting the efficacy of interventions such as PD [psychological debriefing] that often they themselves have developed. Indeed research grants, as well as the livelihoods of individuals employed by companies contracted to provide debriefing services, might depend on it! The last decade has witnessed the emergence of a "disaster industry." (p. 931)

Other scholars have also discussed how high the financial stakes can be in the field of traumatic stress, and how tensions can arise between the goals of clinical science and business (e.g., Gist, Woodall, \& Magenheimer, 1999; Ostrow, 1996).

There are also legal aspects of early intervention. Citing their approach as the "standard of care," Everly and Mitchell (1999, p. 135) have emphasized that by debriefing individuals (e.g., emergency service personnel, firefighters) following severe traumatic events, organizations can reduce risk of law- suits. Everly and Mitchell mentioned examples of people who developed chronic psychological problems after not having been debriefed and successfully sued their employers for negligence. During an interview shortly after the September 11 terrorist attacks, a reporter mentioned to one of us that executives of 80 companies that had offices in the World Trade Center were planning to engage the services of commercial debriefing organizations to prevent PTSD among employees who had survived the attacks. The executives feared lawsuits should they fail to debrief their employees. Ironically, the executives may have had the liability risk backwards. Given the absence of data showing that debriefing works, and given some studies suggesting that debriefing may impede natural recovery from trauma, companies may be at heightened risk if they do debrief their employees, especially if they fail to provide informed consent (i.e., summarize all the studies showing no effect for debriefing). And this liability risk may be especially great if companies simply debrief everyone without conducting a formal psychological assessment first. Of course, debriefing advocates may claim that methodological flaws undermine the probative import of the studies unfavorable to debriefing, so that there is no obligation to tell employees about these studies. However, the lack of convincing empirical support for these interventions remains a serious problem.

Finally, early interventions for trauma, humanitarian in intent, must be understood against background assumptions about psychopathology and suffering in contemporary Western postindustrial society. Intense emotional experience is not necessarily indicative of psychopathology. As Ostrow (1996) observed, the emergency medical services community "may want to reexamine the all-American notion that we should always feel good, that stress is bad and that we have to take corrective action to resolve every negative reaction to stress, even if it is normal" (p. 36).

Acknowledgments-The authors thank the five peer reviewers for their excellent comments on the first draft of this article. The critiques of two reviewers, both leading experts on Critical Incident Stress Debriefing, were especially helpful. We also thank Susan Mineka for her superb editorial guidance. Preparation of this article was supported in part by National Institute of Mental Health Grant MH61268 awarded to Richard J. McNally, by National Health and Medical Research Council Grant 209588 awarded to Richard A. Bryant, and by a Wellcome Trust Principal Research Fellowship awarded to Anke Ehlers.

\section{REFERENCES}

American Psychiatric Association. (1980). Diagnostic and statistical manual of mental disorders (3rd ed.). Washington, DC: Author.

American Psychiatric Association. (1987). Diagnostic and statistical manual of mental disorders (3rd ed., rev). Washington, DC: Author.

American Psychiatric Association. (1994). Diagnostic and statistical manual of mental disorders (4th ed.). Washington, DC: Author.

Amir, M., Weil, G., Kaplan, Z., Tocker, T., \& Witztum, E. (1998). Debriefing with brief group psychotherapy in a homogenous [sic] group of noninjured victims of a terrorist attack: A prospective study. Acta Psychiatrica Scandinavica, 98, 237-242. 
Andrews, B., Brewin, C.R., Rose, S., \& Kirk, M. (2000). Predicting PTSD symptoms in victims of violent crime: The role of shame, anger, and childhood abuse. Journal of Abnormal Psychology, 109, 69-73.

Artiss, K.L. (1963). Human behavior under stress: From combat to social psychiatry. Military Medicine, 128, 1011-1015.

Başoğlu, M., Livanou, M., \& Şalcıoğlu, E. (in press). A single session exposure treatment of traumatic stress in earthquake survivors using an earthquake simulator. American Journal of Psychiatry.

Başoğlu, M., Livanou, M., Şalcıoğlu, E., \& Kalender, D. (in press). A brief behavioural treatment of chronic posttraumatic stress disorder in earthquake survivors: Results from an open trial. Psychological Medicine.

Başoğlu, M., Mineka, S., Paker, M., Livanou, M., \& Gök, Ş. (1997). Psychological preparedness for trauma as a protective factor in survivors of torture. Psychological Medicine, 27, 1421-1433.

Bisson, J.I., Jenkins, P.L., Alexander, J., \& Bannister, C. (1997). Randomised controlled trial of psychological debriefing for victims of acute burn trauma. British Journal of Psychiatry, 171, 78-81.

Blanchard, E.B., Hickling, E.J., Forneris, C.A., Taylor, A.E., Buckley, T.C., Loos, W.R., \& Jaccard, J. (1997). Prediction of remission of acute posttraumatic stress disorder in motor vehicle accident victims. Journal of Traumatic Stress, 10, 215-234.

Bohl, N. (1995, August). Measuring the effectiveness of CISD: A study. Fire Engineering, 148, 125-126.

Bonanno, G.A., \& Kaltman, S. (1999). Toward an integrative perspective on bereavement. Psychological Bulletin, 125, 760-776.

Bonanno, G.A., Wortman, C.B., Lehman, D.R., Tweed, R.G., Haring, M., Sonnega, J., Carr, D., \& Nesse, R.M. (2002). Resilience to loss and chronic grief: A prospective study from preloss to 18-months postloss. Journal of Personality and Social Psychology, 83, 1150-1164.

Bordow, S., \& Porritt, D. (1979). An experimental evaluation of crisis intervention. Social Science and Medicine, 13A, 251-256.

Boscarino, J.A. (1995). Post-traumatic stress and associated disorders among Vietnam veterans: The significance of combat exposure and social support. Journal of Traumatic Stress, 8, 317-336.

Bracken, P.J., \& Petty, C. (Eds.). (1998). Rethinking the trauma of war. London: Free Association Books.

Bramsen, I., Dirkzwager, A.J.E., \& van der Ploeg, H.M. (2000). Predeployment personality traits and exposure to trauma as predictors of posttraumatic stress symptoms: A prospective study of former peacekeepers. American Journal of Psychiatry, 157, 1115-1119.

Breslau, N., Chilcoat, H.D., Kessler, R.C., \& Davis, G.C. (1999). Previous exposure to trauma and PTSD effects of subsequent trauma: Results from the Detroit Area Survey of Trauma. American Journal of Psychiatry, 156, 902-907.

Breslau, N., Chilcoat, H.D., Kessler, R.C., Peterson, E.L., \& Lucia, V.C. (1999). Vulnerability to assaultive violence: Further specification of the sex difference in post-traumatic stress disorder. Psychological Medicine, $29,813-821$.

Breslau, N., Davis, G.C., \& Andreski, P. (1995). Risk factors for PTSD-related traumatic events: A prospective analysis. American Journal of Psychiatry, 152, 529-535.

Breslau, N., Davis, G.C., Andreski, P., \& Peterson, E. (1991). Traumatic events and posttraumatic stress disorder in an urban population of young adults. Archives of General Psychiatry, 48, 216-222.

Brewin, C.R. (2001). Cognitive and emotional reactions to traumatic events: Implications for short-term interventions. Advances in Mind-Body Medicine, 17, 160-196.

Brewin, C.R., Andrews, B., \& Rose, S. (2003). Diagnostic overlap between acute stress disorder and PTSD in victims of violent crime. American Journal of Psychiatry, 160, 783-785.

Brewin, C.R., Andrews, B., Rose, S., \& Kirk, M. (1999). Acute stress disorder and posttraumatic stress disorder in victims of violent crime. American Journal of Psychiatry, 156, 360-366.

Brewin, C.R., Andrews, B., \& Valentine, J.D. (2000). Meta-analysis of risk factors for posttraumatic stress disorder in trauma-exposed adults. Journal of Consulting and Clinical Psychology, 68, 748-766.

Brewin, C.R., Rose, S., Andrews, B., Green, J., Tata, P., McEvedy, C., Turner, S., \& Foa, E.B. (2002). Brief screening instrument for post-traumatic stress disorder. British Journal of Psychiatry, 181, 158-162.

Bryant, R.A. (2000). Cognitive behavior therapy of violence-related posttraumatic stress disorder. Aggression and Violent Behavior, 5, 79-97.

Bryant, R.A., Guthrie, R.M., \& Moulds, M.L. (2001). Hypnotizability in acute stress disorder. American Journal of Psychiatry, 158, 600-604.
Bryant, R.A., \& Harvey, A.G. (1998). Relationship between acute stress disorder and posttraumatic stress disorder following mild traumatic brain injury. American Journal of Psychiatry, 155, 625-629.

Bryant, R.A., \& Harvey, A.G. (2000a). Acute stress disorder: A handbook of theory, assessment, and treatment. Washington, DC: American Psychological Association.

Bryant, R.A., \& Harvey, A.G. (2000b). New DSM-IV diagnosis of acute stress disorder. American Journal of Psychiatry, 157, 1889-1890.

Bryant, R.A., \& Harvey, A.G. (2003). Gender differences in the relationship between acute stress disorder and posttraumatic stress disorder. Australian and New Zealand Journal of Psychiatry, 37, 226-229.

Bryant, R.A., Harvey, A.G., Dang, S.T., Sackville, T., \& Basten, C. (1998). Treatment of acute stress disorder: A comparison of cognitive-behavioral therapy and supportive counseling. Journal of Consulting and Clinical Psychology, 66, 862-866.

Bryant, R.A., Moulds, M.L., Guthrie, R.M., \& Nixon, R.D.V. (2003). Treating acute stress disorder following mild traumatic brain injury. American Journal of Psychiatry, 160, 585-587.

Bryant, R.A., Moulds, M.L., Guthrie, R.M., \& Nixon, R.D.V. (in press). The additive benefit of hypnosis and cognitive behavior therapy in treating acute stress disorder. Journal of Consulting and Clinical Psychology.

Bryant, R.A., Moulds, M.L., \& Nixon, R.D.V. (2003). Cognitive therapy of acute stress disorder: A four-year follow-up. Behaviour Research and Therapy, 41, 489-494.

Bryant, R.A., Sackville, T., Dang, S.T., Moulds, M., \& Guthrie, R. (1999). Treating acute stress disorder: An evaluation of cognitive behavior therapy and supportive counseling techniques. American Journal of Psychiatry, 156, 1780-1786.

Bunn, T.A., \& Clarke, A.M. (1979). Crisis intervention: An experimental study of the effects of a brief period of counselling on the anxiety of relatives of seriously injured or ill hospital patients. British Journal of Medical Psychology, 52, 191-195.

Busuttil, W., Turnbull, G.J., Neal, L.A., Rollins, J., West, A.G., Blanch, N., \& Herepath, R. (1995). Incorporating psychological debriefing techniques within a brief group psychotherapy programme for the treatment of post-traumatic stress disorder. British Journal of Psychiatry, 167, 495502 .

Butler, L.D., Duran, R.E.F., Jasiukaitis, P., Koopman, C., \& Spiegel, D. (1996). Hypnotizability and traumatic experience: A diathesis-stress model of dissociative symptomatology. American Journal of Psychiatry, 153(7, Suppl. 78), 42-63.

Campfield, K.M., \& Hills, A.M. (2001). Effect of timing of Critical Incident Stress Debriefing (CISD) on posttraumatic symptoms. Journal of Traumatic Stress, 14, 327-340.

Carlier, I.V.E., Lamberts, R.D., van Uchelen, A.J., \& Gersons, B.P.R. (1998). Disaster-related post-traumatic stress in police officers: A field study of the impact of debriefing. Stress Medicine, 14, 143-148.

Carlier, I.V.E., Voerman, A.E., \& Gersons, B.P.R. (2000). The influence of occupational debriefing on post-traumatic stress symptomatology in traumatized police officers. British Journal of Medical Psychology, 73, 87-98.

Chemtob, C.M., Tomas, S., Law, W., \& Cremniter, D. (1997). Postdisaster psychosocial intervention: A field study of the impact of debriefing on psychological distress. American Journal of Psychiatry, 154, 415-417.

Conlon, L., Fahy, T.J., \& Conroy, R. (1999). PTSD in ambulant RTA victims: A randomized controlled trial of debriefing. Journal of Psychosomatic Research, 46, 37-44.

Creamer, M.C., O'Donnell, M.L., \& Pattison, P. (in press). The relationship between acute stress disorder and posttraumatic stress disorder in severely injured trauma survivors. Behaviour Research and Therapy.

Dancu, C.V., Riggs, D.S., Hearst-Ikeda, D., Shoyer, B.G., \& Foa, E.B. (1996) Dissociative experiences and posttraumatic stress disorder among female victims of criminal assault and rape. Journal of Traumatic Stress, 9, 253267.

Deahl, M. (2000). Psychological debriefing: Controversy and challenge. Australian and New Zealand Journal of Psychiatry, 34, 929-939.

Deahl, M., Srinivasan, M., Jones, N., Thomas, J., Neblett, C., \& Jolly, A. (2000). Preventing psychological trauma in soldiers: The role of operational stress training and psychological debriefing. British Journal of Medical Psychology, 73, 77-85.

Delahanty, D.L., Herberman, H.B., Craig, K.J., Hayward, M.C., Fullerton, C.S., Ursano, R.J., \& Baum, A. (1997). Acute and chronic distress and 
posttraumatic stress disorder as a function of responsibility for serious motor vehicle accidents. Journal of Consulting and Clinical Psychology, $65,560-567$.

DeLisi, L.E., Maurizio, A., Yost, M., Papparozzi, C.F., Fulchino, C., Katz, C.L., Altesman, J., Biel, M., Lee, J., \& Stevens, P. (2003). A survey of New Yorkers after the Sept. 11, 2001, terrorist attacks. American Journal of Psychiatry, 160, 780-783.

Difede, J., Ptacek, J.T., Roberts, J.G., Barocas, D., Rives, W., Apfeldorf, W.J., \& Yurt, R. (2002). Acute stress disorder after burn injury: A predictor of posttraumatic stress disorder. Psychosomatic Medicine, 64, 826-834.

Dunmore, E., Clark, D.M., \& Ehlers, A. (1999). Cognitive factors involved in the onset and maintenance of PTSD. Behaviour Research and Therapy, 37, 809-829.

Dunmore, E., Clark, D.M., \& Ehlers, A. (2001). A prospective investigation of the role of cognitive factors in persistent Posttraumatic Stress Disorder (PTSD) after physical and sexual assault. Behaviour Research and Therapy, 39, 1063-1084.

Echeburua, E., de Corral, P., Sarasua, B., \& Zubizarreta, I. (1996). Treatment of acute posttraumatic stress disorder in rape victims: An experimental study. Journal of Anxiety Disorders, 10, 185-199.

Ehlers, A., \& Clark, D.M. (2000). A cognitive model of posttraumatic stress disorder. Behaviour Research and Therapy, 38, 319-345.

Ehlers, A., \& Clark, D.M. (2003). Early psychological interventions for adult survivors of trauma: A review. Biological Psychiatry, 53, 817-826.

Ehlers, A., Clark, D.M., Hackmann, A., McManus, F., Fennell, M., Herbert, C., \& Mayou, R.A. (in press). A randomized controlled trial of cognitive therapy, self-help, and repeated assessment as early interventions for PTSD. Archives of General Psychiatry.

Ehlers, A., Mayou, R.A., \& Bryant, B. (1998). Psychological predictors of chronic posttraumatic stress disorder after motor vehicle accidents. Journal of Abnormal Psychology, 107, 508-519.

Engelhard, I.M., van den Hout, M.A., Arntz, A., \& McNally, R.J. (2002). A longitudinal study of "intrusion-based reasoning" and posttraumatic stress disorder after exposure to a train disaster. Behaviour Research and Therapy, 40, 1415-1424.

Everly, G.S., Jr., Boyle, S.H., \& Lating, J.M. (1999). The effectiveness of psychological debriefing with vicarious trauma: A meta-analysis. Stress Medicine, 15, 229-233.

Everly, G.S., Jr., Flannery, R.B., Jr., \& Eyler, V.A. (2002). Critical Incident Stress Management (CISM): A statistical review of the literature. Psychiatric Quarterly, 73, 171-182.

Everly, G.S., Jr., \& Mitchell, J.T. (1999). Critical Incident Stress Management (CISM): A new era and standard of care in crisis intervention (2nd ed.). Ellicott City, MD: Chevron.

Flannery, R.B., Jr. (1999). Critical Incident Stress Management and the Assaulted Staff Action Program. International Journal of Emergency Mental Health, 2, 103-108.

Flannery, R.B., Jr. (2001). The Assaulted Staff Action Program (ASAP): Ten year empirical support for Critical Incident Stress Management (CISD). International Journal of Emergency Mental Health, 3, 5-10.

Flannery, R.B., Jr., Fulton, P., Tausch, J., \& DeLoffi, A.Y. (1991). A program to help staff cope with psychological sequelae of assaults by patients. Hospital and Community Psychiatry, 42, 935-938.

Flannery, R.B., Jr., Hanson, M.A., Penk, W.E., Flannery, G.J., \& Gallagher, C. (1995). The Assaulted Staff Action Program: An approach to coping with the aftermath of violence in the workplace. In L.R. Murphy, J.L. Hurrell, Jr., S.L. Sauter, \& G.P. Keita (Eds.), Job stress interventions (pp. 199-212). Washington, DC: American Psychological Association.

Flannery, R.B., Jr., Hanson, M.A., Penk, W.E., Goldfinger, S., Pastva, G.J., \& Navon, M.A. (1998). Replicated declines in assault rates after implementation of the Assaulted Staff Action Program. Psychiatric Services, 49, 241-243.

Flannery, R.B., Jr., Penk, W.E., \& Corrigan, M. (1999). The Assaulted Staff Action Program (ASAP) and declines in the prevalence of assaults: Community-based replication. International Journal of Emergency Mental Health, 1, 19-21.

Flannery, R.B., Jr., Stone, P., Rego, S., \& Walker, A.P. (2001). Characteristics of staff victims of patient assault: Ten year analysis of the Assaulted Staff Action Program (ASAP). Psychiatric Quarterly, 72, 237-248.

Foa, E.B. (2001). Guidelines for response to the recent tragic events in the US. Unpublished manuscript, University of Pennsylvania, Philadelphia.
Foa, E.B., Ehlers, A., Clark, D.M., Tolin, D.F., \& Orsillo, S.M. (1999). The Posttraumatic Cognitions Inventory (PTCI): Development and validation. Psychological Assessment, 11, 303-314.

Foa, E.B., Hearst-Ikeda, D., \& Perry, K.J. (1995). Evaluation of a brief cognitive-behavioral program for the prevention of chronic PTSD in recent assault victims. Journal of Consulting and Clinical Psychology, 63, 948955.

Foa, E.B., \& Meadows, E.A. (1997). Psychosocial treatments for post-traumatic stress disorder: A critical review. Annual Review of Psychology, 48, $449-480$.

Foa, E.B., \& Rothbaum, B.O. (1998). Treating the trauma of rape: Cognitivebehavioral therapy for PTSD. New York: Guilford Press.

Foa, E.B., Zoellner, L.A., \& Feeny, N.C. (2002). An evaluation of three brief programs for facilitating recovery. Manuscript submitted for publication.

Frank, E., Anderson, B., Stewart, B.D., Dancu, C., Hughes, C., \& West, D. (1988). Efficacy of behavior therapy and systematic desensitization in the treatment of rape trauma. Behavior Therapy, 19, 479-489.

Galea, S., Ahern, J., Resnick, H., Kilpatrick, D., Bucuvalas, M., Gold, J., \& Vlahov, D. (2002). Psychological sequelae of the September 11 terrorist attacks. New England Journal of Medicine, 346, 982-987.

Galea, S., Boscarino, J., Resnick, H., \& Vlahov, D. (in press). Mental health in New York City after the September 11 terrorist attacks: Results from two population surveys. In R.W. Manderscheid \& M.J. Henderson (Eds.), Mental health, United States, 2002. Washington, DC: U.S. Government Print Office.

Gidron, Y., Gal., R., Freedman, S., Twiser, I., Lauden, A., Snir, Y., \& Benjamin, J. (2001). Translating research findings to PTSD prevention: Results of a randomized-controlled pilot study. Journal of Traumatic Stress, 14, 773-780.

Giller, J. (1998). Caring for "victims of torture" in Uganda: Some personal reflections. In P.J. Bracken \& C. Petty (Eds.), Rethinking the trauma of war (pp. 128-145). London: Free Association Books.

Gillespie, K., Duffy, M., Hackmann, A., \& Clark, D.M. (2002). Community based cognitive therapy in the treatment of post-traumatic stress disorder following the Omagh bomb. Behaviour Research and Therapy, 40, 345357

Ginzburg, K., Solomon, Z., \& Bleich, A. (2002). Repressive coping style, acute stress disorder, and posttraumatic stress disorder after myocardial infarction. Psychosomatic Medicine, 64, 748-757.

Gist, R., \& Devilly, G.J. (2002). Post-trauma debriefing: The road too frequently travelled. Lancet, 360, 741-742.

Gist, R., \& Lubin, B. (Eds.). (1999). Response to disaster: Psychosocial, community, and ecological approaches. Philadelphia: Brunner/Mazel.

Gist, R., Woodall, S.J., \& Magenheimer, L.K. (1999). "And then you do the Hokey-Pokey and you turn yourself around." In R. Gist \& B. Lubin (Eds.), Response to disaster: Psychosocial, community, and ecological approaches (pp. 269-290). Philadelphia: Brunner/Mazel.

Gittrich, G. (2003, May 27). \$90 million in Project Liberty mental health funds remains unspent. New York Daily News. Retrieved from http://www. nydailynews.com/05-27-2003/front/story/87199p-79357c.html

Goode, E. (2001, September 16). Some therapists fear services could backfire. The New York Times, p. 21.

Halligan, S., Michael, T., Ehlers, A., \& Clark, D.M. (2003). Posttraumatic stress disorder following assault: The role of cognitive processing, trauma memory, and appraisals. Journal of Consulting and Clinical Psychology, 71, 419-431.

Harvey, A.G., \& Bryant, R.A. (1998a). The effect of attempted thought suppression in acute stress disorder. Behaviour Research and Therapy, 36, $583-590$.

Harvey, A.G., \& Bryant, R.A. (1998b). The relationship between acute stress disorder and posttraumatic stress disorder: A prospective evaluation of motor vehicle accident survivors. Journal of Consulting and Clinical Psychology, 66, 507-512.

Harvey, A.G., \& Bryant, R.A. (1999). The relationship between acute stress disorder and posttraumatic stress disorder: A 2-year prospective evaluation. Journal of Consulting and Clinical Psychology, 67, 985-988.

Harvey, A.G., \& Bryant, R.A. (2000a). Memory for acute stress disorder symptoms: A two-year prospective study. Journal of Nervous and Mental Disease, 188, 602-607.

Harvey, A.G., \& Bryant, R.A. (2000b). Two-year prospective evaluation of the relationship between acute stress disorder and posttraumatic stress disorder following mild traumatic brain injury. American Journal of Psychiatry, 157, 626-628. 
Harvey, A.G., \& Bryant, R.A. (2002). Acute stress disorder: A synthesis and critique. Psychological Bulletin, 128, 886-902.

Herbert, J.D., Lilienfeld, S., Kline, J., Montgomery, R., Lohr, J., Brandsma, L., Meadows, E., Jacobs, W.J., Goldstein, N., Gist, R., McNally, R.J., Acierno, R., Harris, M., Devilly, G.J., Bryant, R., Eisman, H.D., Kleinknecht, R., Rosen, G.M., \& Foa, E. (2001). Psychology's response: Primum non nocere. Monitor on Psychology, 32(11), 4.

Hobbs, M., Mayou, R., Harrison, B., \& Worlock, P. (1996). A randomised controlled trial of psychological debriefing for victims of road traffic accidents. British Medical Journal, 313, 1438-1439.

Holeva, V., Tarrier, N., \& Wells, A. (2001). Prevalence and predictors of acute stress disorder and PTSD following road traffic accidents: Thought control strategies and social support. Behavior Therapy, 32, 65-83.

Horowitz, M.J. (1986). Stress response syndromes: PTSD, grief, and adjustment disorders. Northvale, NJ: Jason Aronson.

Hytten, K., \& Hasle, A. (1989). Fire fighters: A study of stress and coping. Acta Psychiatrica Scandinavica, 80(Suppl. 355), 50-55.

Jenkins, S.R. (1996). Social support and debriefing efficacy among emergency medical workers after a mass shooting incident. Journal of Social Behavior and Personality, 11, 477-492.

Kadet, A. (2002, June). Good grief! Smart Money, 11, 109-114.

Kangas, M., Henry, J.L., \& Bryant, R.A. (in press). The relationship between acute stress disorder and posttraumatic stress disorder following cancer. Journal of Consulting and Clinical Psychology.

Kaplan, Z., Weiser, M., Reichenberg, A., Rabinowitz, J., Caspi, A., Bodner, E., \& Zohar, J. (2002). Motivation to serve in the military influences vulnerability to future posttraumatic stress disorder. Psychiatry Research, 109, $45-49$.

Keane, T.M., Kaufman, M.L., \& Kimble, M.O. (2001). Peritraumatic dissociative symptoms, acute stress disorder, and the development of posttraumatic stress disorder: Causation, correlation or epiphenomena. In L. Sanchez-Planell \& C. Diez-Quevedo (Eds.), Dissociative states (pp. 2143). Barcelona, Spain: Springer-Verlag.

Keane, T.M., Scott, W.O., Chavoya, G.A., Lamparski, D.M., \& Fairbank, J. (1985). Social support in Vietnam veterans with posttraumatic stress disorder: A comparative analysis. Journal of Consulting and Clinical Psychology, 53, 95-102.

Kenardy, J.A., Webster, R.A., Lewin, T.J., Carr, V.J., Hazell, P.L., \& Carter, G.L. (1996). Stress debriefing and patterns of recovery following a natural disaster. Journal of Traumatic Stress, 9, 37-49.

Kessler, R.C., Sonnega, A., Bromet, E., Hughes, M., \& Nelson, C.B. (1995). Posttraumatic stress disorder in the National Comorbidity Survey. Archives of General Psychiatry, 52, 1048-1060.

King, D.W., King, L.A., Foy, D.W., \& Gudanowski, D.M. (1996). Prewar factors in combat-related posttraumatic stress disorder: Structural equation modeling with a national sample of female and male Vietnam veterans. Journal of Consulting and Clinical Psychology, 64, 520-531.

Koopman, C., Classen, C., Cardeña, E., \& Spiegel, D. (1995). When disaster strikes, acute stress disorder may follow. Journal of Traumatic Stress, 8 , $29-46$.

Koopman, C., Classen, C., \& Spiegel, D. (1994). Predictors of posttraumatic stress symptoms among survivors of the Oakland/Berkeley, Calif., firestorm. American Journal of Psychiatry, 151, 888-894.

Koren, D., Arnon, I., \& Klein, E. (1999). Acute stress response and posttraumatic stress disorder in traffic accident victims: A one-year prospective, follow-up study. American Journal of Psychiatry, 156, 367-373.

Lange, A., van de Ven, J.-P., Schrieken, B., \& Emmelkamp, P.M.G. (2001). Interapy: Treatment of posttraumatic stress through the Internet: A controlled trial. Journal of Behavior Therapy and Experimental Psychiatry, $32,73-90$.

Lavender, T., \& Walkinshaw, S.A. (1998). Can midwives reduce postpartum psychological morbidity? A randomized trial. Birth, 25, 215-219.

Lee, C., Slade, P., \& Lygo, V. (1996). The influence of psychological debriefing on emotional adaptation in women following early miscarriage: A preliminary study. British Journal of Medical Psychology, 69, 47-58.

Leeman-Conley, M. (1990, April/May). After a violent robbery . . . Criminology Australia, 1, 4-6.

Litz, B.T., Gray, M.J., Bryant, R.A., \& Adler, A.B. (2002). Early intervention for trauma: Current status and future directions. Clinical Psychology: Science and Practice, 9, 112-134.

Macklin, M.L., Metzger, L.J., Litz, B.T., McNally, R.J., Lasko, N.B., Orr, S.P., \& Pitman, R.K. (1998). Lower pre-combat intelligence is a risk factor for posttraumatic stress disorder. Journal of Consulting and Clinical Psychology, 66, 323-326.

Marshall, G.N., \& Schell, T.L. (2002). Reappraising the link between peritraumatic dissociation and PTSD symptom severity: Evidence from a longitudinal study of community violence survivors. Journal of Abnormal Psychology, 111, 626-636.

Marshall, R.D., Spitzer, R., \& Liebowitz, M. (2000). Dr. Marshall and colleagues reply. American Journal of Psychiatry, 157, 1890-1891.

Marshall, R.D., Spitzer, R., \& Liebowitz, M.R. (1999). Review and critique of the new DSM-IV diagnosis of acute stress disorder. American Journal of Psychiatry, 156, 1677-1685.

Mayou, R.A., Ehlers, A., \& Hobbs, M. (2000). Psychological debriefing for road traffic accidents: Three-year follow-up of a randomised controlled trial. British Journal of Psychiatry, 176, 589-593.

McDonald, J.J., Jr. (2003). Posttraumatic stress dishonesty. Employee Relations Law Journal, 28, 93-111.

McFarlane, A.C. (1989). The aetiology of post-traumatic morbidity: Predisposing, precipitating and perpetuating factors. British Journal of Psychiatry, 154, 221-228

McNally, R.J., \& Shin, L.M. (1995). Association of intelligence with severity of posttraumatic stress disorder symptoms in Vietnam combat veterans. American Journal of Psychiatry, 152, 936-938.

Meichenbaum, D. (1994). A clinical handbook/ practical therapist manual for assessing and treating adults with post-traumatic stress disorder. Waterloo, Ontario, Canada: Institute Press.

Mitchell, J.T. (1983). When disaster strikes ... The Critical Incident Stress Debriefing process. Journal of Emergency Medical Services, 8, 36-39.

Mitchell, J.T. (2002, November 11). CISM research summary. Retrieved from http://www.icisf.org/articles/cism_research_summary.pdf

Mitchell, J.T., \& Everly, G.S., Jr. (2001). Critical Incident Stress Debriefing: An operations manual for CISD, defusing and other group crisis intervention services (3rd ed.). Ellicott City, MD: Chevron.

Murray, J., Ehlers, A., \& Mayou, R.A. (2002). Dissociation and post-traumatic stress disorder: Two prospective studies of road traffic accident survivors. British Journal of Psychiatry, 180, 363-368.

Nishith, P., Mechanic, M.B., \& Resick, P.A. (2000). Prior interpersonal trauma: The contribution to current PTSD symptoms in female rape victims. Journal of Abnormal Psychology, 109, 20-25.

North, C.S., Tivis, L., McMillen, J.C., Pfefferbaum, B., Spitznagel, E.L., Cox, J., Nixon, S., Bunch, K.P., \& Smith, E.M. (2002). Psychiatric disorders in rescue workers after the Oklahoma City bombing. American Journal of Psychiatry, 159, 857-859.

Noyes, R., Jr., \& Kletti, R. (1976). Depersonalization in the face of life-threatening danger: A description. Psychiatry, 39, 19-27.

Noyes, R., Jr., \& Kletti, R. (1977). Depersonalization in response to life-threatening danger. Comprehensive Psychiatry, 18, 375-384.

Nurmi, L.A. (1999). The sinking of the Estonia: The effects of Critical Incident Stress Debriefing (CISD) on rescuers. International Journal of Emergency Mental Health, 1, 23-31.

O'Donnell, M.L., Creamer, M.C., Pattison, P., \& Atkin, C. (2001, December) Traumatic injury: Psychological consequences and their predictors. Paper presented at the meeting of the International Society for Traumatic Stress Studies, New Orleans, LA.

Orner, R.J., \& Schnyder, U. (Eds.). (in press). Reconstructing early intervention after trauma innovations in the care of survivors. Oxford, England: Oxford University Press.

Öst, L.-G., Paunovic, N., \& Gillow, E.-M. (2002). Cognitive-behavior therapy in the prevention of chronic PTSD in crime victims. Manuscript submitted for publication.

Ostrow, L.S. (1996). Critical Incident Stress Management: Is it worth it? Journal of Emergency Medical Services, 21(8), 28-36.

Ozer, E.J., Best, S.R., Lipsey, T.L., \& Weiss, D.S. (2003). Predictors of posttraumatic stress disorder and symptoms in adults: A meta-analysis. Psychological Bulletin, 129, 52-73.

Pennebaker, J.W. (2001). Dealing with a traumatic experience immediately after it occurs. Advances in Mind-Body Medicine, 17, 160-162.

Pennebaker, J.W., \& Beall, S.K. (1986). Confronting a traumatic event: Toward an understanding of inhibition and disease. Journal of Abnormal Psychology, 95, 274-281.

Pennebaker, J.W., \& Harber, K.D. (1993). A social stage model for collective coping: The Loma Prieta earthquake and the Persian Gulf War. Journal of Social Issues, 49, 125-146. 
Perry, S., Difede, J., Musngi, G., Frances, A.J., \& Jacobsberg, L. (1992). Predictors of posttraumatic stress disorder after burn injury. American Journal of Psychiatry, 149, 931-935.

Pitman, R.K., Orr, S.P, Lowenhagen, M.J., Macklin, M.L., \& Altman, B. (1991). Pre-Vietnam contents of posttraumatic stress disorder veterans' service medical and personnel records. Comprehensive Psychiatry, 32, 416-422.

Rachman, S. (2001). Emotional processing, with special reference to post-traumatic stress disorder. International Review of Psychiatry, 13, 164-171.

Raphael, B., \& Dobson, M. (2001). Acute posttraumatic interventions. In J.P. Wilson \& M.J. Friedman (Eds.), Treating psychological trauma and PTSD (pp. 139-158). New York: Guilford Press.

Raphael, B., Wilson, J., Meldrum, L., \& McFarlane, A.C. (1996). Acute preventative interventions. In B.A. van der Kolk, A.C. McFarlane, \& L. Weisæth (Eds.), Traumatic stress: The effects of overwhelming experience on mind, body, and society (pp. 463-479). New York: Guilford Press.

Raphael, B., \& Wilson, J.P. (Eds.). (2000). Psychological debriefing: Theory, practice and evidence. Cambridge, England: Cambridge University Press.

Richards, D. (2001). A field study of critical incident stress debriefing versus critical incident stress management. Journal of Mental Health, 10, 351362.

Riggs, D.S., Rothbaum, B.O., \& Foa, E.B. (1995). A prospective examination of symptoms of posttraumatic stress disorder in victims of nonsexual assault. Journal of Interpersonal Violence, 10, 201-214.

Rose, S., Bisson, J., \& Wessely, S. (2001). Psychological debriefing for preventing post traumatic stress disorder (PTSD) (Cochrane Library, Issue 3). Oxford, England: Update Software.

Rose, S., Brewin, C.R., Andrews, B., \& Kirk, M. (1999). A randomized controlled trial of individual psychological debriefing for victims of violent crime. Psychological Medicine, 29, 793-799.

Rothbaum, B.O., Foa, E.B., Riggs, D.S., Murdock, T., \& Walsh, W. (1992). A prospective examination of post-traumatic stress disorder in rape victims. Journal of Traumatic Stress, 5, 455-475.

Schnurr, P.P., Friedman, M.J., \& Rosenberg, S.D. (1993). Premilitary MMPI scores as predictors of combat-related PTSD symptoms. American Journal of Psychiatry, 150, 479-483.

Schnyder, U., \& Moergeli, H. (in press). The course and development of early reactions to traumatic events: Baseline evidence from a nonintervention follow-up study. In R.J. Orner \& U. Schnyder (Eds.), Reconstructing early intervention after trauma: Innovations in the care of survivors. Oxford, England: Oxford University Press.

Schnyder, U., Moergeli, H., Klaghofer, R., \& Buddeberg, C. (2001). Incidence and prediction of posttraumatic stress disorder symptoms in severely injured accident victims. American Journal of Psychiatry, 158, 594599.

Schuster, M.A., Stein, B.D., Jaycox, L.H., Collins, R.L., Marshall, G.N., Elliott, M.N., Zhou, A.J., Kanouse, D.E., Morrison, J.L., \& Berry, S.H. (2001). A national survey of stress reactions after the September 11, 2001, terrorist attacks. New England Journal of Medicine, 345, 1507-1512.

Schwarz, E.E., Kowalski, J.M., \& McNally, R.J. (1993). Malignant memories: Post-traumatic changes in memory in adults after a school shooting. Journal of Traumatic Stress, 6, 545-553.

Shalev, A.Y. (1992). Posttraumatic stress disorder among injured survivors of a terrorist attack: Predictive value of early intrusion and avoidance symptoms. Journal of Nervous and Mental Disease, 180, 505-509.

Shalev, A.Y. (2000). Stress management and debriefing: Historical concepts and present patterns. In B. Raphael \& J.P. Wilson (Eds.), Psychological debriefing: Theory, practice and evidence (pp. 17-31). Cambridge, England: Cambridge University Press.

Shalev, A.Y., Freedman, S., Peri, T., Brandes, D., \& Sahar, T. (1997). Predicting PTSD in trauma survivors: Prospective evaluation of self-report and clinician-administered instruments. British Journal of Psychiatry, 170, 558-564.

Shalev, A.Y., Freedman, S., Peri, T., Brandes, D., Sahar, T., Orr, S.P., \& Pitman, R.K. (1998). Prospective study of posttraumatic stress disorder and depression following trauma. American Journal of Psychiatry, 155, 630-637.

Shalev, A.Y., Peri, T., Canetti, L., \& Schreiber, S. (1996). Predictors of PTSD in injured trauma survivors: A prospective study. American Journal of Psychiatry, 153, 219-225.

Shalev, A.Y., Peri, T., Rogel-Fuchs, Y., Ursano, R., \& Marlowe, D. (1998). Historical group debriefing after combat exposure. Military Medicine, 163, 494-498.
Silva, R.R., Alpert, M., Munoz, D.M., Singh, S., Matzner, F., \& Dummitt, S. (2000). Stress and vulnerability to posttraumatic stress disorder in children and adolescents. American Journal of Psychiatry, 157, 12291235.

Small, R., Lumley, J., Donohue, L., Potter, A., \& Waldenstrom, U. (2000). Randomised controlled trial of midwife led debriefing to reduce maternal depression after operative childbirth. British Medical Journal, 321, 1043-1047.

Smith, K., \& Bryant, R.A. (2000). The generality of cognitive bias in acute stress disorder. Behaviour Research and Therapy, 38, 709-715.

Solomon, Z., \& Benbenishty, R. (1986). The role of proximity, immediacy, and expectancy in frontline treatment of combat stress reaction among Israelis in the Lebanon War. American Journal of Psychiatry, 143, 613-617.

Solomon, Z., Laor, N., \& McFarlane, A.C. (1996). Acute posttraumatic reactions in soldiers and civilians. In B.A. van der Kolk, A.C. McFarlane, \& L. Weisæth (Eds.), Traumatic stress: The effects of overwhelming experience on mind, body, and society (pp. 102-114). New York: Guilford Press.

Southwick, S.M., Morgan, C.A., Nicolaou, A.L., \& Charney, D.S. (1997) Consistency of memory for combat-related traumatic events in veterans of Operation Desert Storm. American Journal of Psychiatry, 154, 173177.

Spiegel, D. (1996). Dissociative disorders. In R.E. Hales \& S.C. Yudofsky (Eds.), Synopsis of psychiatry (pp. 583-604). Washington, DC: American Psychiatric Press.

Spiegel, D., \& Cardeña, E. (1991). Disintegrated experience: The dissociative disorders revisited. Journal of Abnormal Psychology, 100, 366-378.

Spiegel, D., Classen, C., \& Cardeña, E. (2000). New DSM-IV diagnosis of acute stress disorder. American Journal of Psychiatry, 157, 1890.

Staab, J.P., Grieger, T.A., Fullerton, C.S., \& Ursano, R.J. (1996). Acute stress disorder, subsequent posttraumatic stress disorder and depression after a series of typhoons. Anxiety, 2, 219-225.

Stallard, P., \& Law, F. (1993). Screening and psychological debriefing of adolescent survivors of life: Threatening events. British Journal of Psychiatry, 163, 660-665.

Stroebe, M., Stroebe, W., Schut, H., Zech, E., \& van den Bout, J. (2002). Does disclosure of emotions facilitate recovery from bereavement? Evidence from two prospective studies. Journal of Consulting and Clinical Psychology, 70, 169-178.

Ursano, R.J., Grieger, T.A., \& McCarroll, J.E. (1996). Prevention of posttraumatic stress: Consultation, training, and early treatment. In B.A. van der Kolk, A.C. McFarlane, \& L. Weisæth (Eds.), Traumatic stress: The effects of overwhelming experience on mind, body, and society (pp. 441462). New York: Guilford Press.

van der Kolk, B.A., \& van der Hart, O. (1989). Pierre Janet and the breakdown of adaptation in psychological trauma. American Journal of Psychiatry, $146,1530-1540$.

van Emmerik, A.A.P., Kamphuis, J.H., Hulsbosch, A.M., \& Emmelkamp, P.M.G. (2002). Single session debriefing after psychological trauma: A meta-analysis. Lancet, 360, 766-771.

Vasterling, J.J., Brailey, K., Constans, J.I., Borges, A., \& Sutker, P.B. (1997). Assessment of intellectual resources in Gulf War veterans: Relationship to PTSD. Assessment, 1, 51-59.

Vasterling, J.J., Duke, L.M., Brailey, K., Constans, J.I., Allain, A.N., Jr., \& Sutker, P.B. (2002). Attention, learning, and memory performances and intellectual resources in Vietnam veterans: PTSD and no disorder comparisons. Neuropsychology, 16, 5-14.

Veronen, L.J., \& Kilpatrick, D.G. (1983). Stress management for rape victims. In D. Meichenbaum \& M.E. Jaremko (Eds.), Stress reduction and prevention (pp. 341-374). New York: Plenum.

Wakefield, J.C. (1992). The concept of mental disorder: On the boundary between biological facts and social values. American Psychologist, 47, 373-388.

Wakefield, J.C. (1996). DSM-IV: Are we making diagnostic progress? Contemporary Psychology, 41, 646-652.

Wakefield, J.C., \& Spitzer, R.L. (2002). Lowered estimates-but of what? Archives of General Psychiatry, 59, 129-130.

Warda, G., \& Bryant, R.A. (1998). Cognitive bias in acute stress disorder. Behaviour Research and Therapy, 36, 1177-1183.

Wee, D.F., Mills, D.M., \& Koehler, G. (1999). The effects of Critical Incident Stress Debriefing (CISD) on emergency medical services personnel fol- 
Richard J. McNally, Richard A. Bryant, and Anke Ehlers

lowing the Los Angeles civil disturbance. International Journal of Emergency Mental Health, 1, 33-37.

Wortman, C.B., \& Silver, R.C. (1989). The myths of coping with loss. Journal of Consulting and Clinical Psychology, 57, 349-357.

Yehuda, R. (Ed.). (1999). Risk factors for posttraumatic stress disorder. Washington, DC: American Psychiatric Press.

Yehuda, R. (2002a, March). The lessons of 9-11. Address delivered at the annual meeting of the Anxiety Disorders Association of America, Austin, TX.
Yehuda, R. (2002b). Post-traumatic stress disorder. New England Journal of Medicine, 346, 108-114.

Yehuda, R., \& McFarlane, A.C. (1995). Conflict between current knowledge about posttraumatic stress disorder and its original conceptual basis. American Journal of Psychiatry, 152, 1705-1713.

Yule, W. (1992). Post-traumatic stress disorder in child survivors of shipping disasters: The sinking of the "Jupiter." Psychotherapy and Psychosomatics, 57, 200-205. 